\title{
Die Offenheit für interethnische Freundschaften von Jugendlichen in marginalisierten Wohngebieten
}

\author{
Steffen Zdun
}

Angenommen: 23. Juni 2021 / Online publiziert: 9. Juli 2021

(C) Der/die Autor(en) 2021

Zusammenfassung Obwohl interethnische Freundschaften seit Jahren in der deutschen Soziologie thematisiert werden, handelt es sich meist eher um Defizitdiskurse bzw. eine Beschäftigung mit interethnischen Begegnungen in verschiedenen Settings und deren Auswirkungen auf das Individuum. Hingegen ist die darüber hinausgehende Offenheit für interethnische Freundschaften von Jugendlichen in marginalisierten Stadtteilen eine relativ neue Entwicklung hierzulande und deshalb auch ein empirisch relativ blinder Fleck. Der vorliegende Beitrag widmet sich diesem Trend auf der Datengrundlage einer qualitativen Explorativstudie, in deren Rahmen 45 Jugendliche aus sechs deutschen Städten befragt wurden. Die Ergebnisse geben Einblick in diese Offenheit der Jugendlichen und das Selbstverständnis, mit dem sie in Freundschaften mit lokalen Peers praktiziert wird.

\section{The Openness Towards Interethnic Friendships Among Youths in Marginalized Neighborhoods}

\begin{abstract}
While the meaning of interethnic friendships is addressed by German sociology for years, many accounts focus on individual deficits and/or interethnic encounters in different settings and their impact on the individual. The rather broad openness towards interethnic friendships among youths in marginalized neighborhoods is not just a relatively new development in Germany but therefore also an empirical blind spot in research. This paper addresses this development, based on the results of an explorative qualitative study. 45 youths from six German cities were interviewed. The findings provide insights into the openness of these youths and the implicitness with which it is practiced in friendships with local peers.
\end{abstract}

Steffen Zdun $(\bowtie)$

DJI München, Deutsches Jugendinstitut e. V., Nockherstr. 2, 81541 München, Deutschland

E-Mail: zdun@dji.de 


\section{Einleitung}

Der politische Diskurs in Deutschland (sowie anderen europäischen Ländern) ist seit einigen Jahr(zehnt)en von populistischen Tendenzen durchzogen, die ethnische ${ }^{1}$ Ausgrenzung und Xenophobie beinhalten. Zugleich dokumentiert die empirische Forschung eine teils weit verbreitete Ablehnung anderer Ethnien in ganzen Teilen der Bevölkerung. So sind die viel beachtete Langzeitstudie zu gruppenbezogener Menschenfeindlichkeit (z.B. Heitmeyer 2012, 2018) sowie die sog. „Mitte-Studien“ von Decker und Brähler (2006) bis Zick et al. (2019) nicht nur Grundlage kontroverser Debatten, sondern verdeutlichen seit Jahren die Verbreitung abwertender Einstellungen u.a. gegenüber anderen Ethnien. ${ }^{2}$ Solche Einstellungen finden sich vor allem in der Mittel- und Unterschicht, in verschiedenen Altersgruppen und geschlechterübergreifend.

Zwar gibt es auch weltoffenere Einstellungen bei einem Teil der in solchen Studien Befragten, diese werden aber sowohl in der o.g. als auch in weiteren Forschungen, die vorzugsweise einen Defizitansatz verfolgen bzw. sich ethnischer Homophilie oder der bloßen Zusammensetzung des sozialen Umfeldes widmen (z. B. Janßen und Polat 2006; Kruse und Kroneberg 2019; Leszczensky und Pink 2019; Winkler et al. 2011; Zakikhany 2014) noch immer relativ wenig thematisiert. Zudem liefern Daten zu solchen Einstellungen oder Berechnungen der Kontaktchancen zu anderen Ethnien keine belastbaren Erkenntnisse zur ethnischen Heterogenität oder Homophilie im persönlichen Umfeld bzw. zum Kontakt mit anderen Bevölkerungsgruppen. Dementgegen etabliert sich zusehends ein theoretischer und empirischer Diskurs über sich verändernde Zuschreibungen und Aushandlungen sozialer Grenzen, bei denen teils auch das Ethnische an Bedeutung verliert (z. B. Dannenbeck et al. 1999; Hüttermann 2018; Lamont und Molnár 2002; Schönwalder et al. 2016; Wimmer 2013).

Das im Folgenden verwendete Konzept einer Offenheit für interethnische Freundschaften ist im Spektrum jener Ansätze zu verorten und bündelt verschiedene Aspekte. Vor allem geht es über die bloße Heterogenität bzw. Zusammensetzung der Sozialkontakte hinaus und orientiert sich an der konkreten Interaktionspraxis und Bereitschaft zu interethnischen Kontakten. Es reicht nicht aus, andersethnische Personen zu kennen und im erweiterten Umfeld zu haben, vielmehr geht es um enge und gleichwertige Beziehungen, was diverse Facetten des Denkens und Handelns umfasst. Auf der Grundlage der im Datenmaterial vorliegenden Aspekte werden im

\footnotetext{
1 Zentral für den Terminus ,ethnisch“ sind im Folgenden für Selbst- und Fremdzuschreibungen unterschiedlicher Personenkreise zum einen die nationale Herkunft (der Vorfahren) sowie äußere Merkmale (z. B. Haar- und Hautfarbe). Zum anderen beinhaltet dies die sozialen Konstruktionen, zu denen es bei den Aushandlungen zwischen verschiedenen Akteursgruppen, innerhalb ethnischer communities sowie bei der Bestimmung des eigenen Selbstbildes kommt.

2 Auch wenn der Fokus dieser Studien weitgehend auf Deutschen lag, beschreiben sie gesamtgesellschaftliche bzw. europäische Trends, die sich in ähnlicher Weise auch in ethnischen communities und marginalisierten Wohngebieten hierzulande zeigen, d.h. all dies ist Teil der Lebenswelt der hier untersuchten Jugendlichen. Bedeutsam für die eigene Studie ist, dass sich im Gegensatz bzw. parallel zu diesen Trends Nischen zu bilden scheinen, in denen keine oder weniger Abwertung stattfindet, sondern Offenheit für interethnische Freundschaften besteht.
} 
Folgenden berücksichtigt: Einstellungen zu (m Kontakt mit) anderen Ethnien, Kontaktumfang und -geschehen sowie Interaktionspraxis im Alltag. ${ }^{3}$ Ein solch weiter Fokus auf die sozialen Aushandlungen und die Alltagspraxis dieser Offenheit birgt trotz der o.g. Ansätze immer noch relativ großes Innovationspotenzial - nicht allein in der deutschen Forschungslandschaft.

Eigene empirische Vorarbeiten (z.B. Scholl und Zdun 2013) lieferten hierzu erste Indizien und gaben Anlass für eine vertiefende Untersuchung dieser Offenheit der Jugendlichen in marginalisierten Wohngebieten. ${ }^{4}$ Das Ziel war eine Exploration, wie Offenheit in der Praxis ausgestaltet und ausgehandelt wird. Da sich die bisherigen Erkenntnisse auf qualitative Forschung beschränken, lassen sich noch keine belastbaren Aussagen zur Verbreitung und zum Ausmaß treffen. ${ }^{5}$ Daher stehen im Zentrum der Analyse die Form und das Selbstverständnis, mit dem die Jugendlichen ihre Offenheit für interethnische Freundschaften sowie undoing ethnicity ${ }^{6}$ praktizieren. Letzteres ist ein komplementäres Konzept, welches nicht forschungsleitend war, sich aber für die Auswertung als anschlussfähig erwies.

Die Datengrundlage für diesen Beitrag ist eine qualitative Längsschnittstudie, in der 45 Jugendliche mehr als zwei Jahre ,begleitet“" und in sechs deutschen Städten zu ihrem Freundeskreis befragt wurden. Aufgrund der Omnipräsenz von Narrationen zur Offenheit der Jugendlichen auch über mehrere Befragungszeitpunkte hinaus wurden zwecks Reduzierung der Komplexität hier in die Analyse nur Interviewpassagen aus den Erstgesprächen einbezogen. ${ }^{7}$

Im Folgenden wird zunächst auf den Forschungsstand zu ethnischer Abgrenzung und Homophilie sowie auf den zur Auflösung ethnischer Barrieren eingegangen. Im dritten Kapitel wird das methodische Vorgehen erläutert. Das vierte enthält die empirischen Befunde, die im fünften diskutiert werden.

\footnotetext{
${ }^{3}$ Es wird nicht ausgeschlossen, sondern eher erwartet, dass diese Aspekte durch vertiefende Untersuchungen noch um zusätzliche erweitert werden könnten.

${ }^{4}$ Wir schließen dies in anderen Populationen und Stadtteilen nicht aus, was in größer angelegten, repräsentativen Vergleichsstudien untersucht werden sollte. Die Vorkenntnisse sprechen jedoch dafür, dass diese Entwicklung bislang besonders dort bei jungen Menschen aufzutreten scheint. Deshalb setzte die Untersuchung in einem ersten Schritt zunächst dort an.

5 Obwohl Quantifizierungen ausstehen, ist jedoch davon auszugehen, dass dies nicht nur in den bereits untersuchten Wohngebieten auftritt. Erstens liegen dem Autor inzwischen affirmative Daten aus 20 Wohngebieten aus zehn deutschen Städten (Einwohnerzahl zwischen 50.000 und 3,5 Mio.) vor. Zweitens geht es in den mittlerweile knapp 200 Interviews zur Thematik nicht allein um das soziale Umfeld der Befragten, sondern auch um deren Beobachtungen in eigenen und anderen Wohngebieten. Drittens wird diese Offenheit allerorts in gleicher Weise durch lokale Fachkräfte der Sozialarbeit und Polizei bestätigt.

${ }^{6}$ Dieses Konzept wird in Kap. 3 näher erläutert. Da sich im Diskurs um un/doing die englischen Begriffe etabliert haben, werden diese hier verwendet. Ethnicity steht im Folgenden gleichbedeutend mit dem Begriff des ,Ethnischen“.

7 Veränderungen des sozialen Umfeldes ergaben sich höchstens bzgl. einzelner Personen, aber nicht in Bezug auf die artikulierte Offenheit.
} 


\section{Ethnische Barrieren und Offenheit in marginalisierten Stadtteilen}

\subsection{Ethnische Abgrenzung und Homophilie}

In der deutschsprachigen Forschung wird vielfach postuliert, dass sich die großen ethnischen Minderheiten in marginalisierten Wohngebieten konzentrieren und dort die Eigengruppe präferieren, was u.a. als Segregation (Ceylan 2006; Häußermann und Siebel 2004) oder Etablierung von Parallelgesellschaften (Heitmeyer et al. 1997) bezeichnet wird. Obwohl der starke Zusammenhalt einzelner Populationen teils auch positiv gedeutet wird (Nauck et al. 1997), interpretiert u. a. die kriminalsoziologische Forschung diesen Zusammenhalt sowie die Abgrenzung von anderen Ethnien eher als Risikofaktoren (Karstedt 2000). So werden Menschen mit Migrationshintergrund oft mit sozialer Abschottung und problematischen Verhaltensweisen in Verbindung gebracht. Dies wird teils unzulässig verallgemeinert und es wird der Eindruck vermittelt, dass die Ethnie ursächlich für ein solches Verhalten ist, obwohl bekannt ist, dass es sich hierbei vor allem um eine Folge von sozialer Marginalität handelt (Baier et al. 2009).

Letzteres äußert sich etwa in subjektiv erlebter und objektiv bestehender institutioneller Diskriminierung von Minoritäten, besonders in marginalisierten Stadtteilen (Berger und Kahlert 2005; Hormel und Scherr 2010). Die stadtsoziologische Forschung argumentiert, dass der Rückzug auf die Eigengruppe bei sozial schwachen Populationen zu Perspektivlosigkeit beiträgt sowie zu Frustration infolge von sozialer Ausgrenzung und in territorialem Denken und Gewalt gegenüber Angehörigen und Institutionen der Mehrheitsgesellschaft münden kann (Karstedt 2000; Kurtenbach 2017). Dies ist in verkürzter Form der „Teufelskreis“, in dem Minoritäten in marginalisierten Stadtteilen oftmals aufgrund dichotomer Deutungs- und Analysemuster bis in die Gegenwart gesehen werden.

$\mathrm{Zu}$ all dem liegen seit Jahrzehnten belastbare Erkenntnisse und Theorien vor, die hier nicht grundsätzlich in Frage gestellt werden. Ebenso werden weder sozialer Rückzug noch gesellschaftliche Isolation von Jugendlichen in marginalisierten Wohngebieten abgestritten; diese zeigen sich auch in unseren Daten. Jedoch erscheint es an der Zeit, die Annahme einer ethnischen Homophilie ${ }^{8}$ dieser Jugendlichen auf den Prüfstand zu stellen, zumal deren Wohngebiete nach Vertovec (2007) insbesondere in Großstädten zunehmend ,superdivers“ werden.

Bereits Allport (1954) regte mit seiner Kontakthypothese dazu an, sich stärker mit dem Abbau von Vorurteilen durch den Kontakt mit Minoritäten zu beschäftigen. Dem hat sich nicht zuletzt die Xenophobie-Forschung intensiv gewidmet (z. B. Pettigrew 1998; Schönwalder et al. 2016). Wiederholt konnte repliziert werden, dass nicht jeder Kontakt zum Abbau sozialer Abgrenzung beiträgt, sondern dies besondere Sozialkonfigurationen erfordert. Das Konzept und die Forschung hierzu haben

\footnotetext{
8 Geprägt wurde der Begriff der „Homophilie“ durch Lazarsfeld und Merton (1954). Er thematisiert bei Freundschaften eine Ähnlichkeit der Akteure hinsichtlich des Verhaltens und der Denkweisen. Hierzu kommt es sowohl durch eine selektive Auswahl als auch durch gegenseitige Einflussnahme, d. h. Selektion und Sozialisation (Kandel 1978).
} 
allerdings einen anderen Fokus und sind nur bedingt anschlussfähig für das, worum es bei der Offenheit dieser Jugendlichen für interethnische Freundschaften geht.

Daneben stellen etwa Dubet und Lapeyronnie (1994) ethnische Heterogenität in marginalisierten Stadtteilen insbesondere als Ursache für die Erosion sozialer Kontrolle durch Erwachsene und eine Zunahme von Spannungen zwischen Jugendlichen verschiedener Populationen dar. Andere verweisen auf Konflikte als eine Form der Konkurrenz um knappe Ressourcen (Hohm 2003). Konkurrenz wird hier ähnlich wie bei Millers (1975) Subkulturkonzept stärker im Wettbewerb zwischen verschiedenen Minoritäten verortet als zwischen den „Gewinnern“ und „Verlierern“ einer Gesellschaft.

Sogar in der Netzwerkforschung, welche dezidiert die Heterogenität sozialer Umfelder ergründet, ist meist von weit verbreiteter ethnischer Homophilie die Rede (Janßen und Polat 2006; Kruse und Kroneberg 2019; Leszczensky und Pink 2019; Winkler et al. 2011; Zakikhany 2014). Das hat teils methodische Ursachen, wenn neben der eigenen Ethnie nur nach deutschen Kontakten gefragt wird bzw. der inhaltliche Fokus auf ethnischer Abgrenzung liegt. Es ist aber auch darauf zurückzuführen, dass die hier beschriebene Entwicklung erst seit den 2010er Jahren deutlich in Erscheinung tritt und dies speziell in bestimmten Wohngebieten (s. Fußnote 5), d.h. sie tritt in älteren Datensätzen und anderen Sozialräumen weniger auf. Während Russlanddeutsche z. B. bis vor wenigen Jahren noch meist ,unter sich“ blieben (Zdun 2007), nehmen seitdem die Kontakte Jugendlicher zu anderen Ethnien zu (Scholl und Zdun 2013). Daher beschreiben Fenicia et al. (2010) russlanddeutsche Netzwerke noch als ethnisch homophil und verallgemeinern dies sogar zu einem Verhaltensmuster ethnischer Minderheiten.

Die deutsche Stadtsoziologie hat sich bislang ebenfalls kaum mit dieser Entwicklung beschäftigt. Vielmehr postuliert etwa Schaeffer (2014) weniger Intergruppenkontakte bei steigender ethnischer Diversität. Sutterlüty (2010) verortet negative Zuschreibungen in lokalen Kommunikationsprozessen speziell in multiethnischen Kontexten. Ähnlich verhält es sich bei Citlak et al. (2017), die in einer europäischen Vergleichsstudie konstatieren, dass enge Beziehungen in Stadtteilen mit hoher Diversität meist sozial und ethnisch homophil sind.

Hingegen hat bereits Kandel (1978) auf internationaler Ebene in ihren Arbeiten zu Homophilie in Freundeskreisen darauf hingewiesen, dass deren Ähnlichkeit nicht generell auf festgelegten Merkmalen beruht, sondern diese sich verändern können und es eher darauf ankommt, anhand welcher Kriterien junge Menschen Freund*innen auswählen und in welcher Form sie sich gegenseitig sozialisieren. So wurde bereits die Option eingeräumt, sich hierbei stärker an sozialer Ähnlichkeit, ähnlichen Verhaltensweisen oder an einem geteilten Wohnraum zu orientieren als an ethnischer Herkunft. Entsprechend weisen auch Snyders et al. (1997) und Cairns et al. (1988) darauf hin, dass sich Freundschaften teils aufgrund eines ähnlichen Delinquenzniveaus ergeben und ethnische Belange obsolet werden. Dabei muss es sich nicht einmal um von Beginn an bestehende Ähnlichkeiten der Freundeskreise handeln, sondern die Akteure können sich in einem Lernprozess auch hinsichtlich ihrer Verhaltensweisen einander annähern (Kandel 1978; Prinstein und Dodge 2008). 


\subsection{Auflösung ethnischer Barrieren}

Jahrzehntelang wurden marginalisierte Stadtteile nicht nur seitens der Wissenschaft als segregierte Räume thematisiert, vielmehr dominierte dort tatsächlich ethnische Abgrenzung zwischen den Populationen in Deutschland. Selbst auf gezielte Nachfrage hin, warum man sich nicht weniger in Konkurrenz zueinander sehenkönne und stattdessen eher als eine „Schicksalsgemeinschaft“ mit denselben sozialstrukturellen Defiziten, Lebensbedingungen und Ausgrenzungserfahrungen durch die nicht lokale Bevölkerung, verwiesen Jugendliche verschiedener Ethnien noch in den 2000er Jahren oftmals auf die Andersartigkeit und Fremdheit anderer Bevölkerungsgruppen im eigenen Sozialraum (z. B. Heitmeyer et al. 1997; Zdun 2007).

Inzwischen mehren sich jedoch die Hinweise darauf, dass sich seit den 2010er Jahren in diesen Wohngebieten unter den jungen Menschen zunehmend die Einsicht durchsetzt, dass die gemeinsame Lebenslage und der geteilte Sozialraum bedeutsamer für die Wahl des Freundeskreises sind (Scholl und Zdun 2013; Zdun 2019). Die ethnische Homophilie lässt nach und wandelt sich zu einer Offenheit für interethnische Freundschaften bei gleichzeitiger sozialer Homophilie und einer des geteilten Wohnraums. Von der deutschen Soziologie eher unbeachtet, weist der Erziehungswissenschaftler Reinders schon seit 2004 auf eine ähnliche Entwicklung im Schulsystem hin. Seine Vorarbeiten sind hier aber nur bedingt anschlussfähig wegen seines anderen Fokus: interethnische Kontakte im Schulsystem in „städtischen Räumen industrieller Prägung“ (Reinders 2010, S. 124). Reinders beschäftigt sich demnach mit einer anderen Zielgruppe, deren Mitglieder nicht gemeinsam aufgewachsen sind und kein Wohngebiet teilen, sondern sich vorzugsweise in der Schule und teils noch in der Freizeit treffen. Zudem beschreibt er eine empirische Beobachtung, die sich an Schulen auf bestimmte Jugendliche beschränkt und nicht auf ganze Wohngebiete erstreckt. Des Weiteren verortet er diese ethnische Heterogenität speziell an Hauptschulen, da es dort zu mehr interethnischen Kontakten kommt als an anderen Schultypen. ${ }^{9}$ Das begünstigt in der Folge mehr Freizeitkontakte und mündet in Ko-Kulturation. ${ }^{10} \mathrm{Im}$ vorliegenden Beitrag geht es hingegen zentral um lokal gewachsene Beziehungen.

Obwohl die eigenen Vorkenntnisse mit Reinders in einigen Punkten übereinstimmten, boten seine Ergebnisse eher Hinweise darauf, was es im eigenen Kontext zu explorieren galt. Wenngleich Reinders (2016) nicht die soziale Lage und den Sozialraum betrachtet, konstatiert er ebenfalls, dass Freund*innen stärker anhand ähnlicher Herausforderungen im Leben und persönlicher Identitätsentwicklung ausgewählt werden als anhand der Ethnie. Bei diesen Herausforderungen handelt es sich bei ihm aber nicht um geteilte sozialstrukturelle Defizite, sondern gemeinsames „Jugendlich-Sein“ (Reinders 2010, S. 126) sowie eine „hohe Distanz zur älteren Generation“. Das führt zu „criss-crossing“, d.h. eine kulturelle Abgrenzung von der Familie. „Der kulturelle Habitus andersethnischer Jugendlicher wird dann unter

\footnotetext{
9 Das deckt sich mit Kruses (2017) Ergebnissen, der interethnischen Kontakt ebenfalls weniger an Gymnasien und Gesamtschulen als an Hauptschulen konstatiert.

${ }^{10}$ Dies sind nach Krewer und Eckensberger (1998) „Prozesse der gleichberechtigten Aushandlung gültiger Normen, Werte und Handlungsmuster“ (Reinders 2010, S. 125).
} 
Umständen als Bereicherung in dem Sinne angesehen, dass eine verstärkte Abkehr von der eigenen Herkunftskultur (und damit den Erwachsenen als „Repräsentanten“ dieser Herkunftskultur) im Kontakt mit andersethnischen Peers stattfindet" (Reinders 2004, S. 19). Diese Abkehr ist weniger eine gezielte Provokation der Eltern als ein Element der persönlichen Identitätsentwicklung bzw. ein Schritt der sozialisatorischen Entwicklungsaufgaben (Hurrelmann et al. 2015). Da die Ergebnisse unserer Vorarbeiten auf weitere Motive hindeuteten, ging es in unserer Studie um die Explorierung alternativer Erklärungen solchen Verhaltens.

Es mag überraschen, dass gerade von unserer Zielgruppe eine solche Entwicklung ausgeht. Denn deren Offenheit für interethnische Freundschaften steht den o.g. gesellschaftlichen Tendenzen zu ethnischer Abgrenzung in der Mehrheitsgesellschaft entgegen. Sogar die Eltern der Befragten neigten meist noch zu ethnischer Homophilie (Citlak et al. 2017). Für sie war das Ethnische im Jugendalter ein zentraler Aspekt der sozialen Abgrenzung und für Auseinandersetzungen (Tertilt 1996; Zdun 2007), nicht zuletzt begründet über Konflikte im Herkunftsland. Umso bemerkenswerter ist es, dass die Bedeutung der ethnischen Komponente zwischen den Generationen deutlich nachgelassen zu haben scheint. Während Reinders (2004, S. 135) dies vor allem auf ein im Vergleich zu den Erwachsenen öffentlicheres Freizeitverhalten sowie auf Kontakte in der Schule zurückführt, d.h. mehr interethnischen Kontakt, argumentierte bereits Eisenstadt (1966), dass ,Jugendcliquen und -kulturen [schon immer] als Motor sozialen Wandels" galten. Daher ist es nicht unbedingt erstaunlich, dass sich unsere Zielgruppe dahingehend als innovativ erweist. Zumindest spricht einiges dafür, dass ein solcher Schritt am ehesten von Jugendlichen ausgeht. ${ }^{11}$

Folgt man Kruse (2017) und Reinders (2004), dürften zudem die Rahmenbedingungen in marginalisierten Stadtteilen die Entstehung interethnischer Freundschaften begünstigen. Dafür ist das Ausmaß solcher Kontakte essentiell. Während der gemeinsame Sozialraum früher konfliktfördernd wirkte, könnte er inzwischen regelrecht zu einem Katalysator geworden sein, der infolge großer ethnischer Heterogenität - ebenso wie bestimmte Schul(typ)en - solche Kontakte fördert und ihnen große Bedeutung verleiht. Während Jugendlichen aus Wohngebieten mit geringer ethnischer Heterogenität und Anzahl an Menschen mit Migrationshintergrund nach Reinders meist nur die Schule als Anknüpfungspunkt für interethnischen Kontakt zur Verfügung steht, ${ }^{12}$ sind marginalisierte Wohngebiete vielfach deutlich ethnisch heterogener. In jenen können die Jugendlichen dem sogar teils weder in der Schule noch im öffentlichen Raum entfliehen und treffen ständig auf bekannte Personen, da man mehrere Lebensräume teilt. So zeigen auch Dannenbeck et al. (1999), dass Eigenheiten, Verhaltensweisen, Probleme und Konflikte von Jugendlichen in solchen Wohngebieten nicht grundsätzlich auf ethnische Diversität zurückzuführen sind. Deshalb postulieren sie einen immer wieder unterschiedlichen Umgang mit „Differenzerfahrungen“, d.h. sie sprechen sich gegen rein dichotome Unterscheidungen und Situationsdeutungen aus. Je nach Thematik, Konstellation und Aus-

\footnotetext{
${ }^{11}$ So hat auch Hart (2002) nachgewiesen, dass junge Menschen in jordanischen Flüchtlingslagern trotz einer großen Verbundenheit ihrer Eltern zur Herkunftskultur und Ethnie „Grenzgänge“ unternahmen und sich in ihren sozialen Beziehungen offener für die Ethnie der Ausnahmegesellschaft erwiesen.

12 Mancherorts fallen diese sogar in der Schule gering aus.
} 
gangslage können Alltagserfahrungen somit auf unterschiedliche Weise interpretiert werden, so dass Zuschreibungen und soziale Abgrenzungen anhand von Ethnien an Bedeutung verlieren. Dannenbeck et al. (2001) ergänzen, dass junge Menschen in marginalisierten Wohngebieten zunehmend erkennen, dass ethnische Differenzierungen und Konflikte letzten Endes insbesondere zur Reproduktion der eigenen Marginalität beitragen und interethnischer Zusammenhalt vorteilhafter ist, um dem entgegenzuwirken.

Schönwalder et al. (2016) führten darüber hinaus eine Untersuchung zum $\mathrm{Zu}$ sammenhang von Diversität und Vertrauen in 50 deutschen Wohngebieten durch. Sie bestätigen, dass größere ethnische Heterogenität lokal interethnischen Kontakt begünstigt, berücksichtigen jedoch nicht, wie eng diese Kontakte sind und wie ethnisch heterogen jeweils das persönliche Umfeld ist. Ferner beschäftigen sie sich nicht dezidiert mit marginalisierten Stadtteilen und befragten nur Erwachsene. Dennoch ist ihre Studie einschlägig, weil sie in Anlehnung an Allport (1954) Hinweise dafür liefert, unter welchen Bedingungen sozialräumlich interethnischer Kontakt zunehmen kann, wenngleich das nicht mit einer Offenheit für interethnische Freundschaften gleichzusetzen ist.

Dasselbe gilt für das Konzept des ethnic boundary making, welches in Anlehnung an Barth (1969) und die Erweiterungen durch Lamont und Molnár (2002) dokumentiert, wie bei der Aushandlung sozialer Grenzen der Fokus der Akteure teils von der Ethnie rückt, so dass die Kategorien variieren können, anhand derer in verschiedenen Kontexten und Alltagssituationen soziale Zugehörigkeiten und Abgrenzungen ausgehandelt werden. Nach Wimmer (2013) und Hüttermann (2018) sind diese Grenzen nicht eindeutig zu bestimmen und wechselhaft und lassen Grenzgänge bzw. eine individuelle Verortung in verschiedenen sozialen Kontexten zu, was verschiedene „Mitgliedschaften“ ermöglicht. Deshalb erscheint es empirisch nicht mehr zeitgemäß, ausschließlich mit dichotomen Kategorien in verschiedene soziale Kontexte vorzudringen. Vielmehr bietet es sich an, die soziale Auslotung und Aushandlung lokal bestehender Grenzziehungen und -bewegungen mit einer breiteren Perspektive explorativ zu ergründen.

Nachdem sich bereits in der Feldphase der diesem Aufsatz zugrundeliegenden Interviews eine weitgehende Omnipräsenz der Offenheit für interethnische Freundschaften unter den Befragten abzeichnete, geht es im Folgenden nicht nur darum, dies anhand des Datenmaterials zu dokumentieren. Vielmehr werden der alltägliche Umgang damit sowie die Interaktionspraxis dargestellt.

\section{Methodik}

Um zu untersuchen, inwiefern Jugendliche in marginalisierten Stadtteilen offen für interethnische Freundschaften sind, wurden 45 männliche 14- bis 20-Jährige ${ }^{13}$ aus

\footnotetext{
13 Gewählt wurde das Altersintervall, in dem das Jugendstrafrecht einschlägig ist, da es in der Studie zentral um Jugenddelinquenz ging. Da jene keinen Einfluss auf die festgestellte Offenheit hatte, wird sie hier nicht weiter thematisiert. Auch aufgrund der spezifischen Thematik wurden ausschließlich Männer befragt, da jene stärker als Frauen delinquent werden.
} 
sieben Stadtteilen bzw. sechs Städten in Niedersachsen und Nordrhein-Westfalen (zwischen 50.000 und 600.000 Einwohner*innen) interviewt. ${ }^{14}$ Zwecks Gewährleistung höchster Anonymitätsstandards werden diese Städte nicht genauer benannt. Die Marginalisierung der Wohngebiete wurde anhand von Daten der statistischen Ämter ermittelt. ${ }^{15}$ Einbezogen wurden vorzugsweise Sozialräume, die in Relation zum Gesamtstadtgebiet am meisten marginalisiert waren. Das Ausmaß der jeweiligen Werte variierte zwischen den Städten und Stadtteilen, lag aber überall auf sehr hohem Niveau.

Der ethnische Hintergrund der Befragten variierte ebenfalls, war aber kein Samplingkriterium und dokumentiert lediglich die Vielfalt. Vertreten waren neben keinem (7) folgende Migrationshintergründe: türkisch (13), russisch (7), irakisch (8), ehem. jugoslawisch (4), argentinisch (1), bulgarisch (1), italienisch (1), polnisch (1), südafrikanisch (1) und tunesisch (1). ${ }^{16}$ Die bereits erwähnte Omnipräsenz der Offenheit für interethnische Freundschaften der Jugendlichen verdeutlicht zudem, dass diese Entwicklung nicht auf bestimmte Bevölkerungsgruppen beschränkt ist, und offenbart auch bei Jugendlichen ohne Migrationshintergrund keine Besonderheiten. Dasselbe gilt für die verschiedenen Wohngebiete und Peer-Netzwerke, in denen die Befragungen durchgeführt wurden. Deshalb wird im Ergebnisteil nicht anhand von Herkunft oder Wohngebieten differenziert.

Von allen Interviews wurden nach Einwilligung der Befragten Audioaufnahmen erstellt. Diese wurden zur weiteren Analyse transkribiert und mit altas.ti 7 kodiert. Die Jugendlichen erhielten eine Aufwandsentschädigung, die nicht zuletzt der Motivation zur wiederholten Befragung diente. Bei den Erstinterviews wurde jeweils derselbe Leitfaden verwendet. Die Rekrutierung erfolgte lokal in einschlägigen Jugendtreffs sowie in Jugendheimen und über die Jugendgerichtshilfe. Diese Einrichtungen boten in ihren Räumlichkeiten bzw. inihrer Umgebung eine Plattform für eine gezielte Kontaktaufnahme zu Jugendlichen der Zielgruppe. Die Ansprache erfolgte durch das Projektteam und wurde nicht durch diese Anlaufstellen durchgeführt, um Selektionseffekte durch das Personal zu vermeiden. Jenes diente vielmehr als „Türöffner“ zur Zielgruppe. Befragt wurden ergänzend zehn Fachkräfte aus diesen Institutionen, um zentrale Erkenntnisse zu validieren und zu vertiefen. Die Streuung über verschiedene Institutionen und die Validierung durch Fachkräfte diente zudem dazu, den möglichen Selektionsbias bzgl. der Offenheit für interethnische Freundschaften zu kontrollieren. So waren es lokal nicht bloß einzelne Jugendtreffs, in denen diese Offenheit gelebt wurde, vielmehr erwies sie sich nach Auskunft aller Befragten unisono als integraler Bestandteil des alltäglichen Zusammenlebens der Jugendlichen im Sozialraum. ${ }^{17}$ Der öffentliche Raum, in dem die meiste Freizeit

\footnotetext{
14 Die Befragungen erfolgten von März 2017 bis Mai 2019.

${ }^{15}$ Faktoren waren: Arbeitslosenquote, SGB II-Betroffenheit, Migrant*innenanteil, Haushalte mit Kindern, Ein-Eltern-Familien, durchschnittlicher Mietpreis $/ \mathrm{m}^{2}$.

16 Sämtliche Interviews konnten auf Deutsch geführt werden. Fünf Jugendliche waren in der frühen Kindheit eingewandert und hatten rasch Deutsch gelernt, die anderen waren in Deutschland geboren. Neu Zugewanderte wurden nicht befragt, da es um das hiesige Heranwachsen ging.

17 Zwar gab es lokal vereinzelt Peers, die z.B. aufgrund nationalistischer und ideologischer Einstellungen auf ethnische Homophilie setzten oder aus anderen Gründen allein blieben, diese waren jedoch die Ausnahme.
} 
gemeinsam verbracht wurde, war davon geradezu geprägt. Die Rekrutierung erfolgte dennoch mithilfe der o.g. Institutionen, da deren Räumlichkeiten sich für die Durchführung der Interviews eigneten. Einzelne Interviews wurden auch an anderen Stellen im öffentlichen Raum durchgeführt. Die Maßgabe war hierbei jeweils, dass die Befragten sich einen Ort aussuchen sollten, an dem sie sich möglichst wohl und unbeobachtet fühlten, um das Wohlbefinden und die Auskunftsbereitschaft bei sensiblen Inhalten zu steigern. Der eigenen Forschungserfahrung nach können vertraute Räume und die eigene Wahl des Befragungsortes dazu beitragen.

Die Auswertung erfolgte in Anlehnung an die grounded theory sowohl individuell als auch fallübergreifend. Zudem gingen wir explorativ an die Interviews und die Analyse heran. ${ }^{18}$ Es bestanden keine dezidierten Vorannahmen und kein Vorwissen zur konkreten Praxis der Offenheit für interethnische Freundschaften. Deshalb wurde für deren Erfassung eine problemzentrierte und keine narrative Gesprächsführung präferiert, um gezielte Vertiefungsfragen stellen zu können (Böttger und Strobl 1997).

Für die Auswertung erfolgte für jedes Interview zunächst eine offene Kodierung sowie eine Verknüpfung mit den standardisiert erfassten Daten. ${ }^{19}$ Die erste Analyseeinheit bestand in der Erstellung von Einzelprofilen anhand der Angaben in den Interviews und der Daten des Zusatzfragebogens. So wurde eine möglichst breite Informationsfülle generiert, ohne einen ,data overload“ (Miles und Huberman 1994, S. 56) zu erleiden. Da in den Interviews mit den Fachkräften Nachfragen zu bestimmten Jugendlichen ausgeschlossen waren, wurden die Angaben der Jugendlichen zur Offenheit für interethnische Freundschaften mit den generellen Beobachtungen der Fachkräfte hierzu abgeglichen. Da sich die Angaben der Jugendlichen und der Fachkräfte im Wesentlichen nicht unterschieden, beschränkt sich der Ergebnisteil zwecks besserer Übersichtlichkeit und Vermeidung einer Doppelung der Aussagen auf Zitate der jungen Menschen. Die zweite Analyseeinheit bestand in der fallübergreifenden axialen Kodierung der Interviews mit den Jugendlichen, um zentrale Gemeinsamkeiten und Unterschiede bzw. Muster herauszuarbeiten. Die dritte Analyseeinheit bestand dann in einer Gesamtschau der Ergebnisse, die auf die Einzelfälle zwecks selektiver Kodierung rückgespiegelt wurde (Strauss und Corbin 1990).

In die themenspezifische Analyse floss zudem Hirschauers (2001, 2014) Konzept des undoing ein. Obwohl Hirschauer sich weniger mit ethnicity, sondern vielmehr mit gender und differences beschäftigt und damit einen Gegenentwurf zu West und Zimmermans (1987) doing gender liefert, erwies sich sein Ansatz als anschlussfähig. Hirschauer widerspricht der These, dass es unmöglich ist, spezifische Handlungsformen infolge einer bestimmten sozialen Rolle abzulegen. Vielmehr finde beim undoing ein „Wechsel zu anderen Unterscheidungen statt“ (Hirschauer 2014, S. 183). Im vorliegenden Kontext bedeutet das: Obwohl die Gesellschaft voller ethnischer Unterschiede ist, wird sie nicht gänzlich durch jene geprägt. Menschen können bspw. ethnische Unterschiede gerade dann neutralisieren, wenn diese an Bedeutung verlieren und andere Differenzen wichtiger werden. Nach Hirschauer (2001, S. 215)

\footnotetext{
18 Es bestand lediglich die Vorannahme, dass viele Jugendliche über ethnisch heterogene Freundeskreise verfügen, was allerdings individuell zu überprüfen war.

19 Neben der Soziodemografie wurden die in den vergangenen zwölf Monaten verübten Delikte abgefragt.
} 
geht dadurch die ethnische Identität nicht verloren, sie wird von den Mitmenschen weiterhin wahrgenommen; sie wird jedoch zu einer „Hintergrunderwartung“ und verliert in bestimmten Kontexten an Gewicht als soziale Ordnungskategorie. Bei der Auswertung wurde der Blick deshalb auch auf Aspekte von undoing ethnicity gerichtet. ${ }^{20}$

\section{Ergebnisse}

Bei der vorliegenden Untersuchung handelt es sich um eine explorative Studie, die u. a. darauf abzielt, Grundmuster der Offenheit von Jugendlichen aus marginalisierten Stadtteilen für interethnische Freundschaften zu rekonstruieren. Daher wird im Folgenden vor allem auf drei zentrale Aspekte eingegangen, die sich in den Befragungen als von essentieller Bedeutung erwiesen. Erstens geht es um die Verbreitung und Grenzen dieser Offenheit sowie von Einstellungen zu anderen Ethnien, zweitens um die Verbundenheit zwischen den Jugendlichen, drittens um den alltäglichen Umgang mit ethnischer Heterogenität in Freund- und Partnerschaften.

\subsection{Offenheit für interethnische Freundschaften und deren Grenzen}

Zunächst ist herauszustellen, dass alle interviewten Jugendlichen einen ethnisch heterogenen Freundeskreis beschreiben, welcher die Diversität im eigenen Wohngebiet abbildet und mit dem sie weitgehend von Kindheit an aufgewachsen sind. Dies ist so weit verbreitet und reicht oft so weit, dass die eigene Ethnie nicht die Mehrheit darstellt.

J6: ${ }^{21}$ Ich habe Slowenen, Russen, Türken, Kurdische, Kamerun. Alles Mögliche ist bei mir. (...) Wir sind zusammen groß geworden.

Bedeutsam hieran ist u.a., dass weder in qualitativer noch quantitativer Hinsicht unterschieden wird, mit welcher Ethnie bevorzugt die Freizeit verbracht wird. Man verbringt schlichtweg Zeit mit lokalen Peers, die man spontan trifft oder mit denen man sich verabredet.

J21: Es ist vollkommen egal, wen du triffst. Warum sollte ich mit einem Kurden besser chillen können als mit einem Türken oder Polen? Wichtig ist, wer Zeit hat und Lust, etwas zusammen zu machen.

Insgesamt spielt ethnische Homophilie keine Relevanz mehr im Freundeskreis und stellt somit keine bedeutsame Kategorie für diese Sozialkontakte und deren Aushandlungen dar. Undoing ethnicity erfolgt in dem Sinne, dass die Ethnie unter den Peers als soziale Ordnungskategorie deutlich an Bedeutung einbüßt. Als verbindend wird hingegen das gemeinsame Aufwachsen und Leben in einem Sozialraum

\footnotetext{
20 Zumal hierfür keine konkrete Operationalisierung vorlag, erfolgte dies ebenfalls nicht anhand konkreter Vorannahmen oder Hypothesen. Vielmehr dienten die o.g. Grundannahmen als exploratives Konzept für die Analyse.

21 Es werden die Abkürzungen J für Jugendliche und I für Interviewer*innen verwendet.
} 
dargestellt, das nicht nur Identität stiftet, sondern auch als Rahmen für soziale Abgrenzung von „Auswärtigen“ dient.

J32: Es ist hier egal, welche Herkunft du hast. Wir halten zusammen, weil wir alle von hier sind. (...) Wir haben wenig mit Leuten aus anderen Stadtteilen zu tun und die können wenig mit uns anfangen.

Der Sozialraum erlangt eine zentrale Bedeutung, wohingegen das Ethnische neutralisiert wird. Lokal reicht das so weit, dass die Ethnie nur noch in ganz seltenen Ausnahmefällen zur Abgrenzung fungiert. Vielmehr werden reger Kontakt und sogar enge Freundschaften zu Peers anderer Ethnien als selbstverständlich erachtet.

J2: Ich und meine Freunde sehen keinen Grund, andere Völker auszuschließen.

Wir sind alle Menschen; so sagen wir das untereinander. Um ein guter Freund zu sein, muss einer zu mir passen, mich verstehen und mir zuhören. Man muss sich vertrauen können. Da spielen Länder keine Rolle.

Essentiell scheint vielmehr die Verbundenheit zu sein, die durch den geteilten Sozialraum bzw. das gemeinsame Aufwachsen und die daraus resultierende „Wellenlänge“ entsteht. Daher ist es weniger eine Gegenevidenz, sondern vielmehr eine Facette dieser Offenheit, dass vielfach eine Abgrenzung von Peers aus anderen Sozialräumen, aber teils auch infolge devianter Verhaltensweisen erfolgt. Beispielsweise berichten einige Jugendliche von der irritierenden Erfahrung, zwar im Stadtteil mit allen anderen Ethnien auszukommen, jedoch im öffentlichen Raum gelegentlich auf Geflüchtete zu treffen, die ihnen durch ein sozial nicht angepasstes Auftreten auffallen.

J5: Von uns macht vielleicht auch mal einer den Dicken, wenn er Bock drauf hat, aber eigentlich halten sich alle zurück, zumindest gegenüber Älteren und Fremden. Wir sind ja keine Asis! Deshalb liegt es nicht an der Herkunft, wenn mich Flüchtlinge nerven. Ich bin kein Rassist! Die benehmen sich einfach merkwürdig.

I: Also gibt es doch Menschen, mit denen du schlechter auskommst?

J5: Die Kurden und Iraker, die erst kurz in Deutschland sind. Mit denen haben wir eigentlich so gar nichts zu tun.

I: Wollen die nicht oder ihr?

J5: Wir wollen nicht eigentlich, weil die meisten sind übelst ekelig. Tut mir leid, aber ... Die sind einfach komplett anders, benehmen sich anders, schreien in der Bahn und alles Mögliche.

J41: Eigentlich mag ich Flüchtlinge. Weil meine Tante zum Beispiel selber ein Flüchtling war. Sie kam aus Ungarn und alles. Alles schön und gut. Aber was ich selber nicht mag, ist, wie die neuen Flüchtlinge auf Mädchen zurennen, die angucken, die begrapschen.

Insbesondere das ambivalente Statement von J5 verdeutlicht, dass hier mit zweierlei Maß gemessen wird und es zu einem solchen „Befremden“ nur kommt, wenn es um das Verhalten von Personenkreisen geht, mit denen man sich weniger verbunden fühlt. Sprich, diese Abgrenzung beruht nicht auf der Herkunft der Betreffenden, sondern auf mangelnder Vertrautheit. Dies wird dadurch bekräftigt, dass bestimm- 
te Verhaltensweisen jener als deviant eingestuft werden, wohingegen ein ähnliches Auftreten im Freundeskreis eher (als Ausnahme) neutralisiert wird. Dies widerspricht somit nicht der Offenheit für interethnische Freundschaften. Vielmehr zeigt sich im Sinne von Lamont und Molnár (2002), dass es weiterhin zu sozialer Abgrenzung kommt, jedoch vorzugsweise anhand von abgelehnten Verhaltensweisen bei Personen, mit denen man nicht gemeinsam aufwuchs.

Für den Bedeutungsverlust der ethnischen Dimension sprechen zudem wiederholte Aussagen wie die Folgende:

\section{J29: Mensch ist Mensch!}

Solche Feststellungen dienen den Jugendlichen affirmativ zur intersubjektiven Validierung ihrer Offenheit bzw. der Neutralisierung von ethnicity. Entsprechend erweist sich dies sowohl auf der Einstellungs- als auch der Interaktionsebene im Wohngebiet zwischen den Peers als Selbstverständlichkeit. Sogar auf Nachfrage hin erscheint ihnen weder im Denken noch Handeln eine Differenzierung bzgl. der Ethnie sinnvoll.

J25: Ich könnte gar nicht sagen, was die Unterschiede zwischen den Völkern sein sollen - außer der Sprache, dem Pass und der Religion sind wir gleich.

Türken sind nicht anders als Kurden, als Polen usw.

Aus diesem Selbstverständnis heraus scheint es den Jugendlichen schwer zu fallen, auf Fragen nach den Ursachen für ihre Offenheit anders als mit der Gegenfrage zu reagieren:

\section{J32: Warum sollte das anders sein?}

Zwar ist ihnen bewusst, dass ihre Eltern und ältere Verwandte noch immer ein weitgehend ethnisch homophiles Umfeld pflegen, jedoch reflektieren sie nicht, warum das bei ihnen anders ist. ${ }^{22}$ Diese Unklarheit lässt sich zunächst so deuten, dass es sich um keine bewusste Entscheidung der Jugendlichen für mehr Offenheit für interethnische Freundschaften zu einem bestimmten Zeitpunkt oder infolge konkreter Ereignisse handelt. Vielmehr nehmen diese die ethnische Herkunft im Freundeskreis nicht mehr als adäquates Abgrenzungsmittel wahr und fühlen sich eher mit den lokalen Peers verbunden. In Anlehnung an Dannenbeck et al. (1999) stellt dies einen veränderten Umgang mit „Differenzerfahrungen“ dar, bei dem lokal negative Zuschreibungen und soziale Abgrenzungen anhand von ethnicity an Bedeutung verloren haben. Somit erwiesen sich Fragen dahingehend als ergiebiger, was diese verbindet bzw. was gegen soziale Abschottung spricht. Im Folgenden wird es detaillierter hierum gehen sowie die Praxis dieser Offenheit.

\footnotetext{
22 Selbst berufserfahrenen Fachkräften fehlt es an einer stichhaltigen Erklärung für diesen Wandel, obwohl sie ihn bereits seit einigen Jahren beobachten.
} 


\title{
4.2 Offenheit für interethnische Freundschaften als Ausdruck geteilter Erfahrungen und eines gemeinsamen Wohnraumes
}

Undoing ethnicity findet unter den Befragten nicht allein in einer intersubjektiven Validierung ihrer Offenheit für interethnische Freundschaften statt. In der alltäglichen Praxis verliert dieser Aspekt der Herkunft vielmehr seine Bedeutung als soziale Ordnungskategorie und wird ersetzt durch ein anderes identitätsstiftendes Bindeglied: gemeinsames Aufwachsen im Wohngebiet. Dies beinhaltet: 1. verbindende Erfahrungen, 2. die zeitliche Dauer, die man sich kennt, 3. teils Diskriminierung des Wohngebietes durch Auswärtige sowie 4. damit verbundene soziale Isolation und geringe räumliche Mobilität.

\begin{abstract}
J31: Mit vielen der besten Freunde bin ich seit der Kindheit aufgewachsen und wir haben viel erlebt. Das schweißt zusammen. (...) Natürlich ist da auch, wie Leute aus anderen Gegenden auf uns reagieren und herabschauen. Das schweißt zusammen und sorgt dafür, dass wir gar nicht so viel woanders hingehen. AuBer in der Innenstadt mit den Geschäften gibt es woanders nicht viel mehr zu machen als hier. Aber auch in der Innenstadt werden wir Schwarzköpfe anders angeschaut als die Deutschen. Darauf hat man nicht immer Bock. Aber das bekommt man als Kind noch weniger mit, wo man mit den Freunden zusammenwächst.
\end{abstract}

Die Erlebnisse und Freizeitaktivitäten der Jugendlichen beschränken sich lokal weitgehend auf Allerweltshobbys wie Fußball, Basketball, Kampfsport und gemeinsames „Chillen“ sowie miteinander gut reden zu können und sich zu verstehen alles eher Selbstverständlichkeiten. Es können sogar ganz unterschiedliche Charaktere im Freundeskreis vertreten sein: von sehr aufbrausend bis sehr ruhig. Insgesamt scheint lokal im Hinblick auf Freundschaften fast alles möglich zu sein, d.h. es besteht nicht nur eine Offenheit für interethnische Freundschaften. Zu sozialer Abgrenzung kommt es lokal wie erwähnt höchstens bei störenden Verhaltensweisen wie hohem Delinquenzaufkommen, Drogenkonsum bzw. einem Benehmen in der Öffentlichkeit, das als äußerst abweichend wahrgenommen wird. Diese Ablehnung richtet sich nicht nur gegen Geflüchtete, sondern bei enormer sozialer Abweichung auch gegen bekannte Peers, deren Verhalten, aber nicht deren Ethnie man als störend empfindet.

J12: Mit Freunden muss man sich nur gut verstehen und vertrauen können. Das ist das Wichtigste. Man muss gut reden und chillen können. Oder wir spielen auch mal Fußball.

I: Aber könnten das auch andere Leute aus dem Stadtteil sein?

J12: Ja, es könnten auch andere sein. Es ist eher Zufall, wen ich besser kennengelernt habe und mit wem ich eng geworden bin. Die anderen hier sind auch nicht so anders, aber verbringen halt nicht so viel Zeit zusammen. Nur einzelne frühere Freunde sind so abgedriftet mit Kriminalität und Drogen, dass es gar nicht mehr geht.

Das Konfrontiert-Sein mit denselben Herausforderungen im Leben ergibt sich in den Freundeskreisen zumindest bzgl. einer relativ ähnlichen Lebenslage im So- 
zialraum fast automatisch. Sprich, die Freund*innen teilen aufgrund der lokalen Gegebenheiten weitgehend gewisse sozialstrukturelle Defizite.

J12: Ja, ich würde schon sagen, dass wir uns da alle relativ ähnlich sind. Hier ist keiner komplett arm, aber reiche Familien gibt es auch nicht. Bei den einen gehen die Eltern arbeiten, die anderen gehen zum Amt. Aber davon kann man auch leben. Die Möglichkeiten hier in X (Stadtteil) sind für uns alle gleich, wenn es um Schule, Ausbildung und Arbeit geht.

Wie bei Dannenbeck et al. (2001) beschrieben, scheint es sich inzwischen verbindend zwischen verschiedenen Ethnien auszuwirken, wenn ihnen bewusst wird, dass sie nicht grundsätzlich in Konkurrenz zueinander stehen und interethnischer Zusammenhalt vorteilhafter ist als eine Aufrechterhaltung ethnischer Differenzierungen und Konflikte, welche letzten Endes die Reproduktion der eigenen Marginalität begünstigen.

Somit erscheinen auch lokal, analog zu Reinders (2016) Feststellungen, ähnliche Herausforderungen im Leben und persönliche Identitätsentwicklung prägend bei der Ko-Kulturation. Lokale sozialisatorische und sozialstrukturelle Unwägbarkeiten sind z.B. für viele Jugendliche eine gemeinsame Alltagserfahrung.

J22: Neben gemeinsamen Einstellungen und Erwartungen an Freunde gibt es etwas, was wir nur hier teilen und nicht mit anderen in der Schule. Nur hier verstehen alle, was es bedeutet, in so einer Gegend aufzuwachsen. Klar, wir finden das hier alles okay und normal, aber nicht alles ist gut. Kinder sehen schon Junkies und Drogenhandel. Was uns auch verbindet, ist, dass wir hier gemeinsam durch die Hölle gehen (lacht). Aber wir sprechen darüber und lachen darüber.

So fungiert die gemeinsame Lebenslage als ein essentielles Bindeglied. Deren Bindekraft spiegelt sich insbesondere darin wider, dass sogar Jugendliche, die mit der Familie in benachbarte, sozial bessergestellte Wohngebiete zogen, dort kaum Anschluss suchen und in der Freizeit weiterhin die bisherigen Treffpunkte frequentieren, um den dortigen Peers verbunden zu bleiben. Neue Kontakte werden kaum geknüpft, da man sich anderswo nicht auf einer gemeinsamen Wellenlänge sieht.

I: Hast du Freunde, wo du jetzt wohnst?

J19: Ich kenne da fast niemanden. Eine Person, ein, zwei Leute, aber mehr nicht.

I: Seit wann wohnst du denn da?

J19: Seit zwei Jahren oder so. Aber ich komme nachmittags immer hier hin.

Mit denen dort verstehe ich mich nicht richtig, weil die anders drauf sind. Die leben in einer anderen Welt, haben andere Themen, die sie bewegen.

In Anlehnung an Hirschauer (2014) beinhaltet undoing ethnicity hier somit das doing einer anderen Differenzierung, die auf sozialer und lokaler Zugehörigkeit beruht. Insofern hat sich bei der Wahrnehmung und Aushandlung sozialer Grenzen der Fokus der Akteure weg von der Ethnie und hin zum Zusammenhalt mit lokalen Peers entwickelt (Lamont und Molnár 2002). Demgegenüber bestehen seitens der Jugendlichen im Sinne von sozialer Abgrenzung nur begrenzt Anknüpfungspunkte zu Peers aus sozial bessergestellten Stadtteilen, da zu diesen sowohl eine soziale als 
auch eine lokale Verbundenheit fehlt. Sogar aus einem Schulbesuch in auswärtigen Stadtteilen resultieren entsprechend weitgehend nur bedingt Freundschaften.

J7: Ich gehe ja in X (anderer Stadtteil) in die Realschule, weil es hier keine gibt. Ich chille außerhalb der Schule aber eigentlich nicht mit den Jungs von dort. Es ist nicht, dass wir nicht miteinander klarkommen, aber so richtig eng ist das auch nicht. Das kann man nicht mit hier vergleichen. Die sind schon teils irgendwie okay, aber mehr auch nicht. Irgendwie sind die anders drauf und leben anders.

Wenn überhaupt einer bestimmten Personengruppe, dann spricht man vorzugsweise der Wohnbevölkerung anderer Stadtteile eine fremde „Kultur“ zu, was augenscheinlich auch der Rechtfertigung gegenüber Dritten und vor sich selbst dient, warum diese Kontakte gemieden bzw. nicht vertieft werden. Die starke Grenzziehung anhand dieser Aspekte wird umso deutlicher, wenn man sich die zuvor beschriebene große Diversität in den Peer-Netzwerken der befragten Jugendlichen vergegenwärtigt. Sprich, während jene untereinander über eine enorme Ambiguitätstoleranz verfügen, kommt es hierzu zwecks Abgrenzung kaum bei auswärtigen Peers.

J8: Gerade die aus den besseren Stadtteilen sind manchmal komisch. Die leben auf eine andere Art und können sich das hier gar nicht vorstellen. Wenn man sagt, wo man herkommt, muss man sich anhören: Ist es da nicht gefährlich?

Bemerkenswerterweise lässt sich im Datenmaterial dahingehend keinerlei ethnische Komponente feststellen. Weder mit Blick auf andere Wohngebiete noch mit Blick auf das eigene geht es um diese Kategorie, sondern vielmehr um das Befremden, mit dem man teils auf Auswärtige reagiert bzw. das man bei jenen im gegenseitigen Umgang wahrnimmt. Hier erfolgt ein deutliches doing differences.

Insgesamt scheint das Verbindende der Offenheit für interethnische Freundschaften bei den Jugendlichen zwar größtenteils aus den lokalen Gemeinsamkeiten zu erwachsen, allerdings wird es auch bedingt durch die Abgrenzung von und Ausgrenzung durch Auswärtige, welche deswegen nicht zwangsläufig zum Feindbild werden. Vielmehr zieht man jene weniger als (potenzielle) Freund*innen in Betracht, da der (subjektive) Eindruck konfligierender „Lebenswelten“ besteht.

J31: Verstehen Sie das nicht falsch! Ich habe nichts gegen die Leute aus anderen Gegenden der Stadt, wir haben teils Spaß zusammen in der Schule, aber ganz sind wir nicht auf einer Wellenlänge. So wie die sich anziehen und verhalten, würde hier jeder sofort erkennen, dass die von woanders sind.

\subsection{Thematisierung von Heterogenität trotz Offenheit für interethnische Freundschaften}

Wie Hirschauer (2001) betont, verlieren Aspekte, die beim undoing neutralisiert werden, zwar an Bedeutung als soziale Ordnungskategorie, verschwinden aber nicht zwangsläufig gänzlich aus der sozialen Praxis. Entsprechend wird ethnicity trotz der Offenheit für interethnische Freundschaften in den Interviews teils thematisiert 
oder bewusst umgangen. Eine Option ist es, ethnische Heterogenität in Form von überspitzt formulierten Vorurteilen für Witze auf Kosten anderer zu verwenden. ${ }^{23}$

J11: Also bei mir ist das eigentlich so, also allgemein auch bei jedem aus meiner Klasse, wenn halt irgendwie so ein Pole auftaucht oder ein Türke, dann zählt man halt die ganzen Vorurteile auf, die man über dieses Land kennt und macht sich darüber lustig. Also jetzt zum Beispiel, dass der Türke alles fälscht oder der Pole alles klaut. Wir können darüber lachen, weil wir wissen, dass das nicht stimmt und alle gleich sind.

Ein spielerischer Umgang mit dem Ethnischen ist aber nur eine Möglichkeit der Neutralisierung. Alternativ kann die Herkunft in der Alltagspraxis als unbedeutend erklärt werden.

\section{I: Macht ihr Witze über die Herkunft?}

J16: Damit könnte man höchstens jemand ärgern und das gehört sich unter Freunden nicht. Vor allem wäre das aber auch langweilig und wir machen lieber Scherze über die Person selbst.

J38: Über die anderen Länder will ich nichts wissen. Ich weiß nicht einmal bei allen Freunden, wo die Familie herkommt.

Hierbei handelt es sich um ein bewusstes undoing, um dieser Kategorie keinerlei weitere Bedeutung beizumessen. Eine erweiterte Stufe ist die Vermeidung politischer und religiöser Themen unter den Peers. Obwohl man sich untereinander in anderer Form durchaus provoziert, wird hierauf teils gegenüber bestimmten Peers verzichtet, von denen bekannt ist, dass sie sich bspw. mit Konflikten in den Herkunftsgebieten der Familie besonders identifizieren.

J15: Die Ausnahme ist Politik. Man macht über politische Spannungen keine Witze. Es ist einfacher sich zu mögen, wenn man darauf verzichtet. Dort drauBen gibt es zu viele Probleme. Wir sollten uns da nicht reinziehen lassen und unsere Freundschaft zerstören.

Zwar eignet sich eine solche Vermeidung weniger dazu, interethnische Konflikte $\mathrm{zu}$ überwinden. Allerdings begünstigt sie den Aufbau und die Aufrechterhaltung interethnischer Freundeskreise, was diese Jugendlichen früheren Generationen und anderen Teilen der Gesellschaft in Deutschland durchaus voraushaben, die stärker auf soziale Abgrenzung setzen. Undoing ethnicity in diesem Sinne sieht es vor, dass die Ethnie nicht mehr als Streitthema fungiert, obwohl bzw. gerade weil den Beteiligten die Heterogenität ihres Umfeldes bewusst ist. Dies erfolgt wie beschrieben sogar mit einer individuellen Anpassungsleistung an spezifische Befindlichkeiten.

Da die Eltern der Jugendlichen weiterhin eher ethnisch homophile Beziehungen pflegen, war ferner zu erörtern, inwiefern den Jugendlichen der Spagat zwischen der Familie und dem Freundeskreis gelingt. Im Gegensatz zu Reinders (2004) Erkenntnissen benötigen die interviewten Jugendlichen hierfür kein criss-crossing. Offenheit für interethnische Freundschaften stellt für sie keine kulturelle Abkehr von der Fa-

\footnotetext{
23 Auch in dieser Hinsicht wurden Jugendliche ohne Migrationshintergrund genauso adressiert wie jene mit einem solchen.
} 
milie dar und wird nicht gänzlich getrennt vom Elternhaus praktiziert. Das dürfte auch daran liegen, dass die interethnischen Kontakte nicht erst im Schulsystem mit auswärtigen Peers aufgebaut wurden, sondern von Kindheit an mit lokalen Peers.

J12: Wir wachsen hier halt zusammen auf und kennen uns schon, seit wir Kinder sind. Die Familien kennen sich teils auch oder die älteren Brüder oder Cousins. Mit einigen Freunden bin ich schon seit dem Kindergarten zusammen, andere habe ich erst in der Grundschule kennengelernt.

Entsprechend sind auch die Eltern und Familien der Jugendlichen seit deren Kindheit in diesen Wandel mehr oder weniger einbezogen, auch wenn sie selbst daran kaum partizipieren. Deshalb erfordert die Offenheit der Jugendlichen keine kulturelle Abkehr vom Elternhaus. Vielmehr dürfte es dem Selbstverständnis der Jugendlichen zuträglich sein, dass die meisten Eltern dem wohlwollend gegenüberstehen. Nur wenige Befragte berichten, dass ihre interethnischen Kontakte diesen anfangs oder dauerhaft ein Dorn im Auge sind.

J43: Also ja früher, als ich noch um die zehn war, meinte meine Mutter: Die Kurden sind gefährlich! Halte dich von denen fern! Aber jetzt mit der Zeit sagt meine Mutter auch nichts mehr, weil die Kollegen kommen, essen, trinken bei mir und ich das bei denen. Die sind so wie meine Brüder.

J26: Meine Mama sagt öfters: Lass es mit den Ausländern, mit den Kanaken!

I: Die sagt das heute noch?

J26: Ja, hat sie.

Abgesehen von Einzelfällen unter den Eltern scheint eine relativ breite Akzeptanzhaltung für die Offenheit des Nachwuchses zu bestehen. Denn das zentrale Interesse der Eltern besteht darin, dass die Jugendlichen ,gute“ Freund*innen haben, von denen sie nicht ,runtergezogen“ werden. In Anlehnung an Hart (2002) und Wimmer (2013) können so bislang bestehende Grenzkategorien verwischen, Grenzgänge zwischen Ethnien möglich werden sowie Grenzüberschreitungen und neue soziale „Mitgliedschaften“ sich etablieren.

J23: Meinen Eltern ist das auch egal. Auch wenn das bei denen noch anders lief, stört es sie nicht, dass ich ganz verschiedene Freunde haben. Die interessiert es mehr, dass meine Freunde okay sind und nichts Schiefes machen.

Deshalb benötigen die Befragten im Gegensatz zu Reinders' (2004) Zielgruppe für die Ausgestaltung ihrer interethnischen Kontakte keine alternativen Sozialräume. Das Elternhaus fungiert neben dem öffentlichem Raum und Jugendtreffs teils sogar als Treffpunkt. Dessen Frequentierung beruht vorzugsweise auf der jeweiligen Größe des Wohnraums und der individuellen Gastfreundschaft der Eltern. Insofern wird undoing ethnicity des Nachwuchses seitens der Eltern unterstützt. Und diese überschreiten durch die Kontakte der eigenen Kinder zu andersethnischen Freund*innen ihrerseits ethnische Grenzen.

Nach der Thematisierung der ethnischen Heterogenität im Freundeskreis und der Familie geht es im Folgenden darum, was dies für die ethnische Identität der Jugendlichen bedeutet. Dass Reinders (2010, S. 126) bei interethnischen Freundschaften eine „Überlagerung der ethnischen durch die generationale Identität des Jugend- 
lich-Seins“ konstatiert, könnte auf seine spezielle Zielgruppe zurückzuführen sein. Eine gewisse Abgrenzung vom Elternhaus ist als jugendtypisches Übergangsphänomen in unseren Daten selbstverständlich auch zu finden, aber nicht prägend für die konstatierte interethnische Offenheit. An der ethnischen Identität ändert jene kaum etwas, abgesehen davon, dass man die eigene Identität nicht generell über andere Bevölkerungsgruppen stellt.

J14: Es ist doch egal, welche Herkunft oder welchen Glauben die Menschen haben, weil Mensch ist Mensch!

So scheint die Ko-Kulturation zwar eine Abkehr von ethnisch begründeter Überlegenheit zu begünstigen, aber die Jugendlichen empfinden sich weder als eine Art „Misch-Kultur“ noch als entfremdet von ihrer Herkunft. Dahingehend hat ethnicity als soziale Ordnungskategorie kaum an Bedeutung eingebüßt. Stark verbreitete interethnische Freundschaften sind nicht gleichzusetzen mit einer Gleichgültigkeit gegenüber Aspekten des Ethnischen - sowohl bzgl. der eigenen Identität als auch in Form erlebter Fremdzuschreibungen. Vor allem Letzteres lässt die Offenheit der Jugendlichen so relevant und prägnant erscheinen, weil hierdurch etwas Anderes gelebt und erlebt wird. Zur Offenheit für interethnische Freundschaften und zu undoing ethnicity im Freundeskreis kommt es somit trotz oder evtl. auch gerade wegen andersartiger Alltagserfahrungen mit auswärtigen Peers und der Mehrheitsgesellschaft. Hierdurch werden die Jugendlichen mit ihrer ethnischen bzw. sozialen und lokalen Herkunft wiederholt konfrontiert, weshalb allein schon durch solche Erlebnisse diese Aspekte nie gänzlich an Bedeutung im Alltag verlieren, sondern lediglich im Freundeskreis an Bedeutung einbüßen.

Nur einzelne Jugendliche geben deshalb an, dass ihnen ihre Ethnie weitgehend unwichtig ist.

J11: Ich bin halt Deutscher, aber da denke ich kaum drüber nach. Es ist zwar schön, hier zu leben und von hier zu sein, weil es besser als in anderen Gegenden der Welt ist. Aber das ist es ja nicht, was ich bin, sondern das macht mir nur das Leben leichter.

Dass es hierzu jedoch insbesondere bei Jugendlichen ohne Migrationshintergrund kommt, könnte einerseits an einer ,Umkehr der Mehrheitsverhältnisse“ im Wohngebiet liegen. Andererseits könnte es darauf beruhen, dass jene kaum ethnisch konnotierte, sondern eher soziale Fremdzuschreibungen und Ausgrenzungen erleben und sich deshalb weniger über diesen Herkunftsaspekt definieren.

Die Mehrheit der Befragten tendiert hingegen dazu, sich entweder ausschließlich der „Herkunftskultur“ verbunden zu fühlen, insbesondere wenn dies in der Familie emotional aufgeladen sozialisiert wird, oder sich sowohl dem Herkunftsland der Familie als auch Deutschland verbunden zu fühlen, da man sich als Teil beider Länder empfindet. Entsprechende Grenzgänge und -erfahrungen - speziell mit Blick auf weiterhin eigenethnisch orientierte Eltern - gestalten sich teils ähnlich wie in Harts (2002) Beobachtungen.

J37: Ich sage auch gerne, wenn ich woanders bin, in Albanien quasi zu Besuch, da sagt man nicht: Ich bin Albaner. Ich sage: Ich bin Deutscher, ich komme 
aus Deutschland. So, das ist meine Heimat. Also Albanien ist mein Heimatort, mein Heimatland ist da, wo ich herkomme, wo ich geboren bin, wo ich groß geworden bin. Ich bin in Deutschland groß geworden. Also ich bin in x (andere Stadt) gewesen, wir sind viel umgezogen. Aber da, wo ich ehrlich herkomme und wir auch wieder zurückgekommen sind, das ist einfach unser X (Wohnort).

Ähnlich komplex ist das undoing ethnicity in Partnerschaften. Alle Befragten zeigen sich dahingehend - falls sie Beziehungen eingegangen waren - ebenso ethnisch offen wie im Freundeskreis. Das überrascht insofern nicht, weil die Freundeskreise der interviewten Jugendlichen auch weibliche Peers verschiedener ethnischer Herkunft beinhalten, d.h. auf Freundschaftsebene solche Kontakte zwischen den Geschlechtern Usus sind. Ein Großteil sieht mit Blick auf eine spätere Ehe keine Grenzen, zumal bisherige interethnische Partnerschaften problemlos verliefen oder keine Schwierigkeiten antizipiert werden.

J40: Ich glaube man bekommt ein Gefühl dafür. Also man merkt schon, ob das die richtige Person ist. (...) Also mir ist dann die Religion so was von egal später! Ich hatte schon jetzt verschiedene Freundinnen und das war alles okay. Die Herkunft, die Religion, das kann sich unterscheiden.

Ein Teil der Befragten tendiert mit Blick auf die Ehe hingegen eher zu ethnischer Homophilie. Wie bereits erwähnt, findet die Offenheit der Jugendlichen in Freundschaften nicht zwangsläufig Anwendung in allen Lebensbereichen. Während es zu einer Ablehnung gegenüber auswärtigen Personen etwa eher infolge erfahrener Fremdzuschreibungen und Ausgrenzungen kommt, konnte Homophilie hingegen bei der Heirat rein pragmatische Gründe der Kommunikation und des gegenseitigen Verständnisses in der Familie haben, ohne damit jedoch eine starre Form der Rückbesinnung auf ethnische Homophilie im Erwachsenenalter darzustellen.

J28: Ich will es auch selber so, dass die meine Religion hat und meine Muttersprache spricht. Und nicht so eine andere, die sich nicht einmal mit meinen Eltern verständigen kann. Sie kennt die Nationalität, sie weiß, was man isst, wie man sich verhält, so was. (...) Wenn ich eine Frau nehme, die eine andere Sprache spricht, dann weiß die nicht, wie unser Land halt tickt, wie unsere Familie tickt, wie wir drauf sind.

Das verdeutlicht, dass durch eine Offenheit in Jugendfreundschaften ethnicity keineswegs gänzlich neutralisiert und vergessen wird. Im Wesentlichen verliert sie im Freundeskreis sowie in jugendlichen Paarbeziehungen an Bedeutung; sie kann aber individuell und situativ mit Blick auf eine spätere Ehe reaktiviert werden. Das scheint weniger auf familiärer Kontrolle zu beruhen oder darauf, es den Eltern rechtmachen zu wollen, sondern auf Gemeinschaft, Verbundenheit und Verstehen in der Familie. Denn man sieht sich seitens der Familie weder mit konkreten Forderungen noch mit tradierten Feindbildern konfrontiert. Es geht vielmehr darum, worauf die jungen Menschen mit Blick auf ihr späteres Familienleben am meisten Wert legen. So kann bei starker familiärer Verbundenheit etwa die gemeinsame Sprache zwischen Partner*in und Familie als Kommunikationsgrundlage zum Tragen kommen, wenn die Eltern nicht gut Deutsch sprechen. 
J39: Meine Eltern können nur schlecht Deutsch. Wenn die Frau dann nicht irgendeine Art von Arabisch oder Türkisch kann, dann würde das zu schwer in der Familie. Deshalb soll die besser eine von uns sein.

Lediglich sekundär werden andere Traditionen und kulturelle Denk- und Verhaltensweisen in Erwägung gezogen. ${ }^{24}$ Das gegenseitige Verstehen tangiert somit im wörtlichen Sinne vor allem die Sprache. Dass allerdings in solchen Wohngebieten immer mehr Eltern mit Migrationshintergrund der deutschen Sprache mächtig sind, dürfte interethnische Ehen künftig zusätzlich begünstigen.

Große Bedeutung fällt ethnischer Homophilie in der Ehe im Spezialfall der Jesiden aber noch aus einem weiteren Grund zu. So berichten einzelne Befragte mit einem jesidischen Hintergrund, dass ihnen von klein auf vermittelt wurde, Teil einer verfolgten Population zu sein, deren historisches Überleben daran gebunden ist, intraethnisch zu heiraten.

J41: Diese Religion ist auch sehr selten, es gibt sie nicht so oft. Und es ist so, dass wir auch mal kurz davor waren auszusterben, dass es die Religion mal irgendwann gar nicht gibt. Und warum nicht meine Religion, die ich sehr gerne habe, mal verbreiten? Deswegen würde ich auch gerne meinen Kindern das zumuten, dass sie mal eine Jesidin oder einen Jesiden heiraten.

In solchen Fällen kann undoing ethnicity mit Blick auf die Ehe auf eine große Barriere stoßen, wozu es aber sogar in solchen Populationen, wie Gegenevidenzen im Datenmaterial zeigen, auch nicht zwangsläufig kommen muss. Wie bspw. J26 bestätigt, sind auch in solchen Kontexten Grenzüberschreitungen möglich (siehe auch Hart 2002).

J26: Ich weiß, dass das in manchen jesidischen Familien anders ist, aber meinen Eltern ist es egal, wen ich später heirate. Ich soll bloß glücklich sein.

Insgesamt deutet das Datenmaterial darauf hin, dass ethnische Homophilie mit Blick auf spätere Ehen nicht auszuschließen ist, es hierzu allerdings auch nicht zwangsläufig kommt. So dokumentieren die Daten, dass ethnicity unter den Jugendlichen in marginalisierten Wohngebieten in verschiedener Hinsicht als soziale Ordnungskategorie an Bedeutung eingebüßt hat, aber nicht gänzlich in Vergessenheit geraten ist, teils reaktiviert und thematisiert wird und vor allem bei Aus- und Abgrenzungserfahrungen durch auswärtige Peers virulent bleibt.

\section{Zusammenfassung und Diskussion}

\subsection{Zusammenfassung}

Das ausgewertete Datenmaterial dokumentiert einen weitgehend selbstverständlichen Umgang mit einer Offenheit für interethnische Freundschaften und undoing

\footnotetext{
24 Mit Ausnahme bestimmter Gerichte konnten diese aber nicht einmal konkret benannt werden - ähnlich wie es Befürworter*innen der sog. ,,deutschen Leitkultur“ schwer fällt, jene zu definieren.
} 
ethnicity unter Jugendlichen in marginalisierten Wohngebieten. Diese erklärten, dass Freundschaften für sie keine spezifischen ethnischen Grenzen haben. $\mathrm{Zu}$ sozialer Abgrenzung kam es weniger im Sinne einer Ethnisierung, als vielmehr infolge mangelnder Vertrautheit, weil man nicht gemeinsam aufwuchs und sich nicht auf derselben Wellenlänge sah, vereinzelt auch gegenüber Geflüchteten. Hierbei ging es jedoch weniger um die Herkunft als um abgelehnte Verhaltensweisen von Peers, zu denen kein Zusammengehörigkeitsgefühl bestand, welches essentiell sowohl für die Offenheit als auch den Zusammenhalt unter den interviewten Jugendlichen war. Entsprechend wurden teils Verhaltensweisen von Geflüchteten zwecks Abgrenzung als deviant beschrieben, wohingegen ein ähnliches Benehmen von Freund*innen und anderen lokalen Peers neutralisiert wurde, so lange diese ein gewisses Maß an Delinquenz nicht überschritten.

Ursächlich für die Offenheit für interethnische Freundschaften und ein Grundpfeiler des undoing ethnicity scheint insbesondere, dass aus Sicht der Jugendlichen nichts dagegen, aber einiges dafür spricht. Eine Verbundenheit bestand insbesondere zwischen lokalen Peers, die sich weitgehend von früher Kindheit an kannten und zahlreiche Lebens- sowie Ausgrenzungserfahrungen durch Peers aus sozial bessergestellten Wohngebieten teilten. Dabei waren es weniger Besonderheiten als Alltäglichkeiten sowie die Dauerhaftigkeit und Regelmäßigkeit des Kontakts, die zusammenschweißten. Die Diversität der Freundeskreise umfasste neben der Ethnie und dem Geschlecht auch unterschiedliche Persönlichkeiten der Peers sowie den Umgang mit Andersartigkeit. Letzteres erfolgte einerseits als Neutralisierung von ethnicity in Form von Thematisierung, Desinteresse oder Ausblendung bzw. Ignorierung zumindest von Streitthemen zwischen Ethnien. Andererseits hatte man sich aneinander gewöhnt, unterschiedliche Charaktere kennen und damit umzugehen gelernt. In dieser Hinsicht wurde Diversität bewusst gelebt und nicht vermieden oder abgelehnt. Lediglich äußerst abweichende Verhaltensweisen unter lokalen Peers stießen auf Ablehnung und führten zu Ausgrenzung.

Außerdem erfolgte eine soziale Abgrenzung bzw. ergaben sich kaum Anknüpfungspunkte zu Peers aus sozial bessergestellten Wohngebieten, die anders oder zumindest nicht gemeinsam mit den Interviewten aufgewachsen sind, weniger sozialstrukturelle Defizite kannten und teils mit Vorbehalten, negativen Fremdzuschreibungen und Ängsten auf die Sozialräume der Jugendlichen reagierten. Diesen gegenüber fand ein doing sozialer und lokaler Zugehörigkeit statt.

Von der elterlichen Kultur wendeten sich die Jugendlichen nicht generell ab zumindest benötigten sie keine speziellen Räume, um ihre interethnischen Freundschaften zu pflegen. Vielmehr waren sowohl der öffentliche Raum und Jugendtreffs von zentraler Bedeutung als auch das Elternhaus, in dem anders-ethnische Freund*innen bis auf wenige Ausnahmen willkommen waren.

Beim ethnischen Selbstbild der Jugendlichen handelte es sich eher nicht um eine „Misch-Kultur“, vielmehr konnte dieses sich unterschiedlich gestalten. Weder die Offenheit für interethnische Freundschaften noch undoing ethnicity führten somit dazu, dass etwas Neues entstand oder ethnicity für die eigene Identitätsbildung generell an Bedeutung verlor. Vielmehr fand im Freundeskreis ein Miteinander statt, das als gelebte Diversität zu bezeichnen ist. Gleichzeitig fühlte sich die Mehrheit der Jugendlichen entweder der eigenen Ethnie verbunden oder teils der eigenen Eth- 
nie und teils als deutsch. In ein ethnisches Kategoriendenken sowie entsprechende Selbst- und Fremdzuschreibungen wurden sie im Alltag eher durch auswärtige Peers und die Mehrheitsgesellschaft gedrängt.

Bedeutsam ist, dass diese Offenheit nicht nur unter den interviewten männlichen Peers bestand, sondern im Freundeskreis auch zwischen männlichen und weiblichen Jugendlichen zum Tragen kam. Dies zeigt sich auch in jugendlichen Paarbeziehungen. Lediglich bzgl. einer späteren Ehe äußerten sich einzelne Jugendliche weniger offen. Ursächlich dafür waren antizipierte Probleme bei der Kommunikation mit der Familie, vor allem bei deutschen Sprachdefiziten der Eltern, sowie im Spezialfall einzelner Jesiden. Letztere tendierten bei einer Heirat zumindest aus aktueller Sicht zu einer traditionellen Rückbesinnung mit dem Ziel der „Aufrechterhaltung“ ihrer politisch verfolgten Ethnie. Abgesehen davon schien es eine weitgehend individuelle Entscheidung zu sein, ob eine interethnische Ehe in Betracht gezogen und mehr Wert auf zwischenmenschliche Belange gelegt wurde als auf sprachliche und kulturelle.

\subsection{Diskussion}

Obwohl die bisher vorliegenden Daten weder repräsentativ für die einbezogenen noch für sämtliche marginalisierten Stadtteile in Deutschland sind, besteht Grund zu der Annahme, dass die hier vorgelegten Erkenntnisse sich nicht ausschließlich auf die untersuchten Orte beschränken. Es liegen inzwischen über 200 bestätigende Interviews mit Jugendlichen aus 20 Wohngebieten aus zehn Städten vor, die seit den 2010er Jahren geführt wurden; hinzu kommen knapp 50 mit lokalen Fachkräften. Aufgrund der einhellig bestätigten Verbreitung der geschilderten Entwicklung sowie affirmativer Aussagen von Fachkräften aus weiteren Städten ist zu postulieren, dass es hierzu auch in anderen Sozialräumen kommt. Dennoch wird nicht davon ausgegangen, dass es sich um eine flächendeckende Entwicklung in allen marginalisierten Wohngebieten in Deutschland handeln muss. Einerseits ist nicht auszuschließen, dass bestimmte Populationen weiterhin recht stark auf ethnische Homophilie setzen, z.B. Angehörige der sog. ,,arabischstämmigen Clans“, oder (noch) keinen sozialen Anschluss finden, z. B. Teile der Geflüchteten. Andererseits ist davon auszugehen, dass die Offenheit für interethnische Freundschaften unter Jugendlichen bspw. anhand des lokalen Ausmaßes an ethnischer Heterogenität, des Anteils an Menschen mit Migrationshintergrund sowie des Grades der Marginalisierung variieren kann. Das spricht für weitere komparative Studien zur Thematik und vor allem für Quantifizierungen.

In den vorliegenden Daten haben sämtliche Befragten die aus ihrer Sicht bestehende lokale Selbstverständlichkeit einer solchen Offenheit und von undoing ethnicity unter den Jugendlichen bestätigt. Es erscheint daher an der Zeit, dieser Entwicklung seitens der Soziologie mehr Beachtung zu schenken, u. a. in Form vertiefender Untersuchungen, der Erhebung repräsentativer Daten sowie Theoriebildung. Nicht zuletzt dürfte sich eine weitere Erforschung der Ursachen lohnen, auch in Wohngebieten, die bzgl. ethnischer Heterogenität und des Anteils an Menschen mit Migrationshintergrund stärker variieren. Berücksichtigt werden sollten zudem Jugendliche aus sozial bessergestellten Sozialräumen - nicht allein als Vergleichsgruppe, vielmehr zur Untersuchung von deren Offenheit für interethnische Freundschaften sowie un/ 
doing ethnicity. In Anlehnung an Wimmer (2013) und Hüttermann (2018) wird es insbesondere bei sozialen Vergleichen darauf ankommen, nicht in vorgefertigten dichotomen Kategorien verhaftet zu bleiben, sondern unvoreingenommen zu explorieren, anhand welcher Kriterien es lokal zur Praxis und Aushandlung von Grenzgängen, -erfahrungen und -überschreitungen kommt sowie ggf. zu verschiedenen „Mitgliedschaften“ in unterschiedlichen sozialen Kontexten.

Hierbei wird es sich lohnen, sich an die Vorarbeiten von Reinders (2004, 2010, 2016) anzulehnen, der sich in Deutschland als ein Pionier in diesem Bereich erwiesen hat. Infolge seiner andersgelagerten empirischen Perspektive und Zielgruppe ist er zu teils alternativen Einsichten und Erklärungen gelangt, welche künftig ggf. stärker mit den hier dargestellten Erkenntnissen verbunden werden können. Speziell das Konzept der Ko-Kulturation könnte sich als zielführend erweisen, da sich auch im eigenen Datenmaterial ähnliche Aushandlungsprozesse zwischen Jugendlichen dazu nachweisen ließen, wie miteinander umgegangen wird und welche Handlungsmuster akzeptabel sind. Zudem könnte sein Konzept des criss-crossing neben anderen Motiven bzw. Formen einer Offenheit für interethnische Freundschaften genauer untersucht werden, um das Spektrum insgesamtexakter zu bestimmen. So widerspricht die von ihm thematisierte Abkehr von der elterlichen Kultur nicht unseren Erkenntnissen, sondern ist vielmehr als Gegenevidenz zu sehen, da von verschiedenen Varianten der Interaktionspraxis auszugehen ist - insbesondere bei Berücksichtigung unterschiedlicher Zielgruppen. Zum einen gilt es sich diesen Zielgruppen empirisch stärker zu widmen. Zum anderen gibt es in der soziologischen Theoriebildung bislang insbesondere eine Beschäftigung mit doing ethnicity sowie undoing gender und doing differences (Hirschauer 2001, 2014). Im Bereich des undoing ethnicity bestehen noch größere Lücken; sogar bei der Operationalisierung.

Darüber hinaus würde es sich im Kontext von undoing ethnicity sowie doing differences lohnen, detaillierter zu untersuchen, an welchen Stellen, aus welchen Gründen und mit welchen Dynamiken andere Personenkreise abgelehnt und ausgegrenzt werden. So zeigte sich hier etwa, dass Geflüchtete infolge negativer Erlebnisse und mangelnder Vertrautheit, d.h. keines gemeinsamen Aufwachsens, sowie Peers aus sozial besser gestellten Stadtteilen abgelehnt wurden oder zumindest kaum soziale Anknüpfungspunkte bestanden. Dies kann als doing sozialer und lokaler Zugehörigkeit eingestuft werden, aber auch als Reaktion auf Erfahrungen von sozialer Ausgrenzung. ${ }^{25}$

Obwohl das gemeinsame Aufwachsen und die Verbundenheit der Jugendlichen in den marginalisierten Wohngebieten von zentraler Bedeutung für deren interethnische Freundschaften gewesen zu sein scheint, waren hierfür ebenfalls die teils andersartigen, negativen Alltagserfahrungen mit auswärtigen Peers und der Mehrheitsgesellschaft von Bedeutung. Sprich, wer in seinem Freundeskreis interethnische Offenheit praktizieren kann, aber in anderen sozialen Kontexten daran gehindert wird und negative Fremdzuschreibungen erfährt, kann zusätzlich dazu motiviert werden, sich von auswärtigen Peers abzugrenzen und sich lokalen Peers verbunden zu fühlen. In welchem Maße es hierzu jedoch kommt, ist eine weitere Forschungslücke, bei deren

25 Einschlägig und beachtenswert bzgl. eines Zusammenhalts infolge gemeinsamer Ausgrenzungserfahrungen erscheinen ebenfalls die Arbeiten von Mecheril und Hoffarth (2009) und Ottersbach et al. (2014). 
Bearbeitung auch die Erkenntnis von Dannenbeck et al. (2001) aufgegriffen werden kann, dass marginalisierte Jugendliche untereinander ggf. auf interethnischen Zusammenhalt setzen, um der sozialen Reproduktion ihrer Marginalität entgegenzuwirken. Es wäre also interessant zu ergründen, inwiefern tatsächlich ein solches Bewusstsein besteht und wie es zur Abgrenzung von Personen genutzt wird, von denen man sich sozial ausgegrenzt fühlt.

Zudem regen die vorliegenden Erkenntnisse zur Untersuchung von langfristigen Entwicklungen an. Da die untersuchte interethnische Offenheit eine relativ neue Entwicklung in Deutschland ist, wird sie noch kaum von lokalen Erwachsenen praktiziert - teils tendieren sogar noch ältere Geschwister der Jugendlichen zu ethnischer Homophilie. Demnach scheint diese Entwicklung bislang eher die Altersgruppe bis zu den Heranwachsenden zu betreffen. Deshalb ist unklar, in welche Richtung sich dies im Erwachsenenalter entwickeln wird, ob es sich in der nächsten Generation fortsetzt und welche Dynamiken all dies annimmt. So stellt sich die Frage, ob sich die Jugendlichen dahingehend nach Eisenstadt (1966) fortlaufend als Motor sozialen Wandels erweisen werden. ${ }^{26}$

Ferner würde es sich lohnen, detaillierter und langfristig zu ergründen, wie sich diese Entwicklung auf Eheschließungen in marginalisierten Stadtteilen auswirkt. Generell spricht für eine auch in dieser Hinsicht relativ große Offenheit, dass die Ethnie in jugendlichen Paarbeziehungen ebenfalls deutlich an Bedeutung verloren zu haben scheint und sogar für das Erwachsenenalter von einem ganzen Teil der Befragten als eher unwichtig antizipiert wurde. Das heißt, auch hierzeigen die interviewten Jugendlichen mehr Offenheit als die Gesamtgesellschaft. Denn repräsentative Vergleichsstudien konstatieren bis in die Gegenwart weitgehend, dass es sowohl auf Seiten von Aufnahmegesellschaften als auch von ethnischen Minderheiten Ressentiments gegenüber interethnischen Beziehungen gibt, was u.a. mit unterschiedlichen familiären Werten, Geschlechtervorstellungen und Religiosität erklärt wird (Carol 2016). Dennoch verdeutlicht das Datenmaterial, und das wird empirisch langfristig $\mathrm{zu}$ verfolgen sein, dass und nach welchen Kriterien ein Teil der jungen Menschen sich bei Eheschließungen für ethnische Homophilie entscheidet. Auch wenn nicht grundsätzlich davon auszugehen ist, dass bei diesen Personen die Offenheit für interethnische Sozialkontakte im Erwachsenenalter gänzlich verloren geht, da zumindest die Offenheit für solche Freundschaften bestehen bleiben dürfte, ist die Kontextabhängigkeit von Offenheit und undoing ethnicity weiter zu ergründen. Vertiefungen in dieser Hinsicht, sowohl biografisch mit Blick auf den Lebensverlauf als auch situativ in verschiedenen Lebensbereichen, können dazu beitragen, der Vielfalt und Mehrdimensionalität dieser Entwicklung empirisch gerecht zu werden. Hierbei könnte sich u.a. auch Wimmers (2013) Unterscheidung zwischen verbalen Distinktionsmustern und gelebter sozialer Praxis als zielführend erweisen. Dabei handelt es sich jedoch nur um ein plausibles Konzept, welches Anwendung finden könnte, weshalb an dieser Stelle abermals empirische Offenheit der Forschenden anzumahnen ist,

\footnotetext{
${ }^{26}$ Um dies nicht bloß abzuwarten, könnten bereits jetzt zumindest Befragungen von Kindern in marginalisierten Wohngebieten durchgeführt werden, um zu untersuchen, ob diese zumindest ebenfalls eine solche Offenheit und undoing ethnicity praktizieren.
} 
um explorativ der vollen Tragweite der sozialen Auslotung und Aushandlung lokal bestehender Grenzziehungen und -bewegungen gerecht zu werden.

Davon abgesehen sollte ethnische Homophilie in der Ehe auch nicht grundsätzlich und ausschließlich als Anzeichen von „Rückständigkeit“ thematisiert werden, zumal diese zahlreiche Ursachen haben kann. Wenn es etwa um die Kommunikation in der Familie geht bzw. die Aufrechterhaltung der eigenen Kultur und Ethnie, ist das keineswegs gleichzusetzen mit sozialer Ab- und Ausgrenzung, insbesondere wenn die Betreffenden ansonsten weiterhin eine interethnische Offenheit im Freundeskreis praktizieren. Das macht die Kontextabhängigkeit dieser Entwicklung so bedeutsam, die sich zuletzt auch bei den Sozialkontakten mit Peers aus anderen Wohngebieten zeigt.

Darüber hinaus sind einerseits weder eine Offenheit für interethnische Freundschaften noch undoing ethnicity damit gleichzusetzen, dass die eigene Herkunft aufgegeben wird, wenn man im Sinne von Ko-Kulturation denkt. Denn die Umsetzung dieser Offenheit und undoing ethnicity kann auch darin bestehen, ethnische Heterogenität zuzulassen, damit die Ko-Kulturation nicht in einem undefinierbaren Gemisch aus Normen und Handlungsmustern mündet (Krewer und Eckensberger 1998). Andererseits bedeutet weder eine solche Offenheit noch undoing ethnicity, dass die Jugendlichen und deren Familien ihre ethnische Herkunft aufgeben, wenn sie bspw. die deutsche Sprache erlernen. Denn ohne Deutschkenntnisse wären die Kommunikationsmöglichkeiten im Alltag eingeschränkt und damit die Chancen, gleichberechtigt an der Gesellschaft zu partizipieren (ebd.).

In der Stadtsoziologie und anderen Fachsektionen werden ethnische Minderheiten in marginalisierten Wohngebieten noch weitgehend mit ethnischer Homophilie in Verbindung gebracht. Eine Offenheit für interethnische Freundschaften wird, wenn überhaupt, nur punktuell thematisiert und nicht als eine sich zunehmend etablierende Lebensweise. Solche Diskurse beschränken sich weitgehend auf interethnischen Kontakt, ohne dessen Interaktionspraxis genauer zu erörtern - selbst in Kontexten mit großer ethnischer Diversität bleibt dies eher oberflächlich (s. Abschn. 2.1) ${ }^{27}$ Deshalb wäre auch hierzulande stärker an Kandels (1978) Erkenntnis anzuknüpfen, dass sich die Selektion von Freund*innen im Jugendalter an diversen Kriterien der Ähnlichkeit orientieren kann, wodurch das Ethnische teils obsolet wird. Entsprechend könnte es sich ebenfalls lohnen, in künftigen Untersuchungen stärker das Konzept des ethnic boundary making in Anlehnung an Barth (1969) sowie Lamont und Molnár (2002) zu berücksichtigen, um den Fokus darauf zu richten, wie bei der Aushandlung sozialer Grenzen, Zugehörigkeiten und Abgrenzungen Kategorien wie ethnicity an Bedeutung verlieren und durch andere Kategorien ersetzt werden.

Insgesamt zeigt sich ein relativ großer Forschungsbedarf $\mathrm{zu}$ interethnischen Freundschaften und Kontakten in dem Bereich, der sich gegen eine Problemperspektive wendet und die alltäglichen Aushandlungen und die Praxis einer dahingehenden Offenheit in den Fokus rückt. Dies ist bedeutsam, weil in diesem Kontext nicht nur bereits bekannte Themen mit einer neuen Perspektive untersucht werden

\footnotetext{
27 Eine Ausnahme ist Schwiertz (2019) Studie zur Selbstorganisation von geflüchteten Jugendlichen in Deutschland, in der es um die damit verbundene Praxis dieser Offenheit geht. Hierbei handelt es sich jedoch um ein deutlich anderes Themenfeld.
} 
können, sondern weil eine neue soziale Entwicklung zumindest bei Jugendlichen in marginalisierten Stadtteilen parallel zu weit verbreiteten populistischen Tendenzen in der Gesellschaft gezeigt werden kann.

Funding Open Access funding enabled and organized by Projekt DEAL.

Open Access Dieser Artikel wird unter der Creative Commons Namensnennung 4.0 International Lizenz veröffentlicht, welche die Nutzung, Vervielfältigung, Bearbeitung, Verbreitung und Wiedergabe in jeglichem Medium und Format erlaubt, sofern Sie den/die ursprünglichen Autor(en) und die Quelle ordnungsgemäß nennen, einen Link zur Creative Commons Lizenz beifügen und angeben, ob Änderungen vorgenommen wurden.

Die in diesem Artikel enthaltenen Bilder und sonstiges Drittmaterial unterliegen ebenfalls der genannten Creative Commons Lizenz, sofern sich aus der Abbildungslegende nichts anderes ergibt. Sofern das betreffende Material nicht unter der genannten Creative Commons Lizenz steht und die betreffende Handlung nicht nach gesetzlichen Vorschriften erlaubt ist, ist für die oben aufgeführten Weiterverwendungen des Materials die Einwilligung des jeweiligen Rechteinhabers einzuholen.

Weitere Details zur Lizenz entnehmen Sie bitte der Lizenzinformation auf http://creativecommons.org/ licenses/by/4.0/deed.de.

\section{Literatur}

Allport, Gordon. 1954. The nature of prejudice. Cambridge: Addison-Wesley.

Baier, Dirk, Christian Pfeiffer, Julia Simonson, und Susann Rabold. 2009. Jugendliche in Deutschland als Opfer und Täter von Gewalt. Erster Forschungsbericht zum gemeinsamen Forschungsprojekt des Bundesministeriums des Innern und des KFN. Hannover: KFN.

Barth, Fredrik T. 1969. Ethnic groups and boundaries. The social organization of culture difference. Bergen, Oslo, Tromsö: Universitesforlaget.

Berger, Peter, und Heike Kahlert (Hrsg.). 2005. Institutionalisierte Ungleichheiten. Wie das Bildungswesen Chancen blockiert. Weinheim: Juventa.

Böttger, Andreas, und Rainer Strobl. 1997. Rekonstruktion und Fremdverstehen im qualitativen Interview. In Differenz und Integration, Hrsg. Karl-Siegbert Rehberg, 308-311. Opladen: Budrich.

Cairns, Robert B., Beverley D. Cairns, Holly J. Neckerman, Scott D. Gest, und Jean-Louis Gariepy. 1988. Social networks and aggressive behavior: peer support or peer rejection? Developmental Psychology 24:815-823.

Carol, Sarah. 2016. Social integration and intermarriage in Europe: islam, partner choices and parental influence. New York: Routledge.

Ceylan, Rauf. 2006. Ethnische Kolonien. Entstehung, Funktion und Wandel am Beispiel türkischer Moscheen und Cafés. Wiesbaden: VS.

Citlak, Banu, Sebastian Kurtenbach, Megan Lueneburg, und Meglena Zaltkova (Hrsg.). 2017. The new diversity of family life in Europe. Wiesbaden: VS.

Dannenbeck, Clemens, Felicitas Eßer, und Hans Lösch. 1999. Herkunft (er)zählt. Befunde über Zugehörigkeiten Jugendlicher. Münster: Waxmann.

Dannenbeck, Clemens, Felicitas Eßer, und Hans Lösch. 2001. An-, Zu- und Ungehörigkeiten Jugendlicher: Herkunft als Auskunft? In Unterschiedlich verschieden. Differenz in der Erziehungswissenschaft, Hrsg. Helma Lutz, Norbert Werning, 231-248. Opladen: Leske + Budrich.

Decker, Oliver, und Elmar Brähler. 2006. Vom Rand zur Mitte - Rechtsextreme Einstellungen und ihre Einflussfaktoren in Deutschland. Berlin: Friedrich-Ebert-Stiftung.

Dubet, Francois, und Didier Lapeyronnie. 1994. Im Aus der Vorstädte. Der Zerfall der demokratischen Gesellschaft. Stuttgart: Klett-Cotta.

Eisenstadt, Shmuel N. 1966. Modernization: Protest and Change. Englewood Cliffs: Prentice-Hall.

Fenicia, Tatjana, Markus Gamper, und Michael Schönhuth. 2010. Integration, Sozialkapital und soziale Netzwerke. Egozentrierte Netzwerke von (Spät-)Aussiedlern. In Knoten und Kanten. Soziale Netzwerkanalyse in Wirtschafts- und Migrationsforschung, Hrsg. Markus Gamper, Linda Reschke, 305-332. Bielefeld: transcript. 
Hart, Jason. 2002. Children and nationalism in a Palestinian refugee camp in Jordan. Childhood 9:35-47.

Häußermann, Hartmut, und Walter Siebel. 2004. Stadtsoziologie. Eine Einführung. Frankfurt a.M.: Campus.

Heitmeyer, Wilhelm (Hrsg.). 2012. Deutsche Zustände. Frankfurt a.M.: Suhrkamp.

Heitmeyer, Wilhelm. 2018. Autoritäre Versuchungen. Frankfurt a.M.: Suhrkamp.

Heitmeyer, Wilhelm, Joachim Müller, und Helmut Schröder. 1997. Verlockender Fundamentalismus: Türkische Jugendliche in Deutschland. Frankfurt a.M.: Suhrkamp.

Hirschauer, Stefan. 2001. Das Vergessen des Geschlechts. Zur Praxeologie einer Kategorie sozialer Ordnung. Kölner Zeitschrift für Soziologie und Sozialpsychologie 41:208-235.

Hirschauer, Stefan. 2014. Un/doing Differences. Die Kontingenz sozialer Zugehörigkeiten. Zeitschrift für Soziologie 43:170-191.

Hohm, Hans-Jürgen. 2003. Urbane soziale Brennpunkte, Exklusion und soziale Hilfe. Opladen: Budrich.

Hormel, Ulrike, und Albert Scherr (Hrsg.). 2010. Diskriminierung, Grundlagen und Forschungsergebnisse. Wiesbaden: VS.

Hurrelmann, Klaus, Ulrich Bauer, Matthias Grundmann, und Sabine Walper (Hrsg.). 2015. Handbuch Sozialisationsforschung. Weinheim: Juventa.

Hüttermann, Jörg. 2018. Figurationsprozesse der Einwanderungsgesellschaft. Zum Wandel der Beziehungen zwischen Alteingesessenen und Migranten in deutschen Städten. Bielefeld: transcript.

Janßen, Andrea, und Ayça Polat. 2006. Soziale Netzwerke türkischer Migrantinnen und Migranten. Aus Politik und Zeitgeschichte 47:11-17.

Kandel, Denise B. 1978. Homophily, selection, and socialization in adolescent friendships. The American Journal of Sociology 84:427-436.

Karstedt, Susanne. 2000. Social dynamics of crime and control. New theories for a world in transition. Oxford: Oxford University Press.

Krewer, Bernd, und Lutz H. Eckensberger. 1998. Selbstentwicklung und kulturelle Identität. In Handbuch der Sozialisationsforschung, Hrsg. Klaus Hurrelmann, Dieter Ulich, 573-594. Weinheim: Beltz.

Kruse, Hanno. 2017. Close neighbors, separate lives: an investigation of residential barriers and bridges to the social integration of young immigrants in Germany. Mannheim: Universität Mannheim.

Kruse, Hanno, und Clemens Kroneberg. 2019. More than a sorting machine: ethnic boundary making in a stratified school system. American Journal of Sociology 125:431-484.

Kurtenbach, Sebastian. 2017. Leben in herausfordernden Wohngebieten. Das Beispiel Köln-Chorweiler. Wiesbaden: VS.

Lamont, Michèle, und Virág Molnár. 2002. The study of boundaries across the social sciences. Annual Review of Sociology 28:167-195.

Lazarsfeld, Paul F., und Robert K. Merton. 1954. Friendship as a social process: a substantive and methodological analysis. In Freedom and control in modern society, Hrsg. Morroe Berger, Theodore Abel, und Charles H. Page, 18-66. New York: Van Nostrand.

Leszczensky, Lars, und Sebastian Pink. 2019. What drives ethnic Homophily? A relational approach on how ethnic identification moderates preferences for same-ethnic friends. American Sociological Review 84:394-419.

Mecheril, Paul, und Britta Hoffarth. 2009. Adoleszenz und Migration. Zur Bedeutung von Zugehörigkeitsordnungen. In Adoleszenz - Migration - Bildung, Hrsg. Vera King, Hans-Christoph Koller, 239-258. Wiesbaden: VS.

Miles, Matthew B., und Michael Huberman. 1994. Qualitative data analysis. An expanded sourcebook. Thousand Oaks: SAGE.

Miller, Walter. 1975. Violence by youth gangs and youth groups as a crime problem in major American cities. Washington: US Government Printing Office.

Nauck, Bernd, Annette Kohlmann, und Heike Diefenbach. 1997. Familiäre Netzwerke, intergenerative Transmission und Assimilationsprozesse bei türkischen Migrantenfamilien. Kölner Zeitschrift für Soziologie und Sozialpsychologie 49:477-499.

Ottersbach, Markus, Sabine Roeber, Lisa Rosen, Sultan Schulz, und Miriam Yildiz. 2014. ,Ostheim ist einfach, cok güzel ya. Manche sagen Ostheim ist asozial oder so, aber das ist alles Blöff“. In Migration, Stadt und Urbanität, Hrsg. Thomas Geisen, Christine Riegel, und Erol Yildiz, 217-235. Wiesbaden: VS.

Pettigrew, Thomas F. 1998. Intergroup contact theory. Annual Review of Psychology 49:65-85.

Prinstein, Mitchell J., und Kenneth A. Dodge. 2008. Current issues in peer influence research. In Understanding peer influence in children and adolescents, Hrsg. Mitchell J. Prinstein, Kenneth A. Dodge, 3-13. New York: Guilford. 
Reinders, Heinz. 2004. Subjektive Statusgleichheit, interethnische Kontakte und Fremdenfeindlichkeit bei deutschen Jugendlichen. Zeitschrift für Soziologie der Erziehung und Sozialisation 24:182-202.

Reinders, Heinz. 2010. Peers und Migration - zur Bedeutung von inter- und intraethnischen Peerbeziehungen im Jugendalter. In Freundschaft, Cliquen und Jugendkulturen, Hrsg. Marius Harring, Oliver Böhm-Kasper, Carsten Rohlfs, und Christian Palentien, 123-140. Wiesbaden: VS.

Reinders, Heinz. 2016. Interethnische Peer- und Freundschaftsbeziehungen. In Handbuch Peerforschung, Hrsg. Sina-Mareen Köhler, Heinz-Hermann Krüger, und Nicole Pfaff, 237-248. Opladen: Budrich.

Schaeffer, Merlin. 2014. Ethnic diversity and social cohesion: immigration, ethnic fractionalization and potentials for civic action. Burlington: Ashgate.

Scholl, Jennifer, und Steffen Zdun. 2013. Gute Freunde und gute Freunde sind nicht das Gleiche. Zur Bedeutung von Freundschaftsbeziehungen in frühen Phasen von desistance männlicher Heranwachsender. Soziale Probleme 24:213-244.

Schönwälder, Karen, Sören Petermann, Jörg Hüttermann, Steven Vertovec, Miles Hewstone, Dietlind Stolle, Katharina Schmid, und Thomas Schmitt. 2016. Diversity and contact. London: Palgrave Macmillan.

Schwiertz, Helge. 2019. Migration und radikale Demokratie: politische Selbstorganisierung von migrantischen Jugendlichen in Deutschland und den USA. Bielefeld: transcript.

Snyder, James, Ellen Horsch, und Jocelyn Childs. 1997. Peer relationships of young children: affiliative choices and the shaping of aggressive behavior. Journal of Clinical Child Psychology 26:145-156.

Strauss, Anselm, und Juliet Corbin. 1990. Basics of qualitative research: grounded theory procedures and techniques. Beverly Hills: SAGE.

Sutterlüty, Ferdinand. 2010. In Sippenhaft: Negative Klassifikationen in ethnischen Konflikten. Frankfurt a.M.: Campus.

Tertilt, Hermann. 1996. Turkish power boys. Frankfurt a.M.: Suhrkamp.

Vertovec, Steven. 2007. Super-diversity and its implications. Ethnic and Racial Studies 30:1024-1054.

West, Candace, und Don H. Zimmerman. 1987. Doing gender. Gender and Society 1:125-151.

Wimmer, Andreas. 2013. Ethnic boundary making: institutions, power, networks. Oxford: Oxford University Press.

Winkler, Niels, Annabell Zentarra, und Michael Windzio. 2011. Homophilie unter guten Freunden: Starke und schwache Freundschaften zwischen Kindern mit und ohne Migrationshintergrund. Soziale Welt $62: 25-43$.

Zakikhany, Corinna. 2014. Ethnische Netzwerke, soziale Integration und soziale Ungleichheit: Bedingungen und Auswirkungen der Einbindung von Migranten in ethnische Netzwerke. Eine Netzwerkanalyse. Gießen: Universität Gießen.

Zdun, Steffen. 2007. Ablauf, Funktion und Prävention von Gewalt: Eine soziologische Analyse der Verhaltensweisen in den Cliquen junger Russlanddeutscher. Frankfurt a.M.: Peter Lang.

Zdun, Steffen. 2019. The fluid nature of street culture: non-violent participation, changes in adult life, and crumbling ethnic barriers in Germany. European Journal of Crime, Criminal Law and Criminal Justice 27:207-225.

Zick, Andreas, Beate Küpper, und Wilhelm Berghan. 2019. Verlorene Mitte - Feindselige Zustände. Bonn: Dietz. 